\title{
The Role of Forced Oscillators of Coupled Circuits in Radiation Physics: New Linear Accelerator Design Improving Tomo-Scanning Technology (Radiotherapy and CT), Heisenberg-Euler Scatter, and Extension to Bremsstrahlung with GeV Electrons
}

\author{
W. Ulmer \\ Gesellschaft Qualitätssicherung in der Medizin, Deizisau and MPI of Physics, Göttingen, Germany \\ Email: waldemar.ulmer@gmx.net
}

How to cite this paper: Ulmer, W. (2021) The Role of Forced Oscillators of Coupled Circuits in Radiation Physics: New Linear Accelerator Design Improving Tomo-Scanning Technology (Radiotherapy and CT), Heisenberg-Euler Scatter, and Extension to Bremsstrahlung with $\mathrm{GeV}$ Electrons. Journal of Applied Mathematics and Physics, 9, 707-735.

https://doi.org/10.4236/jamp.2021.94051

Received: March 4, 2021

Accepted: April 18, 2021

Published: April 21, 2021

Copyright $\odot 2021$ by author(s) and Scientific Research Publishing Inc. This work is licensed under the Creative Commons Attribution International License (CC BY 4.0).

http://creativecommons.org/licenses/by/4.0/

\begin{abstract}
The quantization of circuits has received to be rather attractive in domains of solid state-molecular-and biophysics, since the quanta referred to as Q-bits play a significant role in the design of the quantum computer and entangled structures. Quantized circuits cannot be applied without modifications, since the energy differences are not equidistant and the polarization of the excited states has to be accounted for having particular importance for the creation of virtual states. Applications of the presented theory are scanning methods in radiotherapy without multi-leaf collimators, which may be realized in tomo-scanning radiotherapy and in the $\mathrm{keV}$ domain, which provides a new design of CT. The problem of lateral scatter in the target and energy storage by heat production is significantly reduced by a multilayer system with focusing the impinging electrons at the walls and by a magnetic field. The verification of the Heisenberg-Euler scatter of crossing beams of $9 \mathrm{MV}$ is a central problem of photon physics and can be solved by the new bremsstrahlung technique. A comparison with GEANT 4 Monte-Carlo data indicates that the presented method also works in the $\mathrm{GeV}$ domain, and a multi-target can improve the bremsstrahlung yield. GEANT 4 provides the spatial distribution, whereas the virtual oscillator states only show the created energy spectrum. In every case, the exploitation yield can be drastically improved by the superiority of the focused multitarget system compared to a single standard target, and the door to new technologies is opened.
\end{abstract}




\section{Keywords}

Harmonic Oscillators in Charge Space, Polarization of Energy Levels, Bremsstrahlung Creation, Focusing of Multitargets, Reduction of Scatter and Heat Production

\section{Introduction}

The forced circuit oscillator in quantum theory, which contains the addition term of the form $U(t)=U_{0} \cdot \mathrm{e}^{i \cdot \omega \cdot t}$, is significant in many disciplines of pure and applied physics, such as quantum electrodynamics, molecular and biophysics inclusive biorhythms [1] [2] [3] [4] [5], design of the quantum computers [6] [7] as well as in disciplines of classical electrotechnics, e.g., control theory, design of networks, information technology [8]. The quantum theoretical treatment of the mentioned physical subject appears to be rather attractive, namely the description of molecular and nuclear processes by circuits. However, an essential deficiency is that the quantized oscillator in the charge space as well as in the position space implies equidistant energy levels, given by

$E_{n}=(n+1 / 2) \cdot \hbar \omega_{0}(n=0,1,2, \cdots)$. A glance at atomic/molecular, solid state or nuclear physics indicates that constant energy differences between the eigen-states do, in general, not exist. In the present study, the polarization of the dielectric constant $\varepsilon$ implying $\varepsilon(\omega)$ and entering the capacitor is accounted for. By that, the equal distances of the energy levels are removed, and, above all, the polarization increases with increasing oscillator energy superimposed by the external forces. This behavior is rather important to describe the creation of bremsstrahlung, but even in the low-energy domain, we cannot neglect the polarization influences of the energy levels. In problems of classical physics, the forced oscillator implies further severe problems, if the force frequency $\omega$ agrees with the oscillator eigen-frequency $\omega_{0}$, and only a resistance term can overcome this problem. The translation of the resistance problem to quantum mechanics is not a trivial one, since the uncertainty relation must not be violated, and a nonlinear term in the Schrödinger equation must be accounted for [9] [10] [11] [12].

\section{Methods}

Abbreviations: Inductivity of a coil: $L$; mutual magnetic coupling between coils. M; Capacitor. $C$; mutual electric coupling $C_{I}$ by common dielectrics $\varepsilon$. If $\mathcal{E}=1$ : vacuum or air with $C_{0}=C$; in the presence of the electric coupling the capacitor reads $C=C_{0}\left(1+C_{I}\right)$. However, by virtue of the time-dependence of $U_{0}(t)$ the dielectric factor $\varepsilon$ assumes $\varepsilon>1$ and may become a function of the frequency $\omega_{0}$, i.e.: $\varepsilon=\varepsilon\left(\omega_{0}\right)$. The creation of bremsstrahlung can be founded by coupled harmonic oscillator processes, where besides the mutual magnetic coupling the electric coupling plays a significant role, since the role of virtual orbitals (oscillator states) is closely connected to the polarizability of the dielectric properties. 
The method even works for bremsstrahlung up to $9 \mathrm{GeV}$.

The following denominations and explanations used in Figure 1 are used in this study:

Figure 1 shows the basic principle in this study, two coupled circuits are driven by the oscillator forces $U_{1} \cdot \mathrm{e}^{i \omega t}$ and $U_{2} \cdot \mathrm{e}^{i \omega t}$. In addition to the usually mutual magnetic coupling $M$ the electric coupling $C_{I}$ is included, which may assume either positive or negative values. The special case without electric and magnetic coupling and $U_{1}=U_{2}=U_{0}$ yields two independent circuits. The indices of the charge can be omitted, and in classical electrotechnics Formula (1) plays a significant role:

$$
\left.\begin{array}{l}
L \cdot \ddot{Q}+\frac{1}{C_{0}} \cdot Q=U_{0} \cdot e^{i \cdot \omega \cdot t} \\
\rightarrow \ddot{Q}+\omega_{0}^{2} \cdot Q=\frac{U_{0}}{L} \cdot \mathrm{e}^{i \cdot \omega \cdot t}
\end{array}\right\}
$$

The well-known eigenfrequency of this oscillator is given by:

$$
\omega_{0}^{2}=\frac{1}{L \cdot C_{0}}
$$

With the help of the "ansatz" $Q=Q_{0} \cdot \mathrm{e}^{i \cdot \omega \cdot t}$ the solution of Equation (1) assumes the shape:

$$
Q_{0}=\frac{1}{\omega_{0}^{2}-\omega^{2}} \cdot \frac{U_{0}}{L}
$$

As well-known, Equation (1) yields Equation (1a), if $\omega=\omega_{0}$ and may only be overcome by an additional damping term, which in electrotechnics is an $\mathrm{Ohm}$ resistance R. An immediate translation of Equation (1) by taking account for damping to QM might require a nonlinear Schrödinger equation in order to incorporate damping as already mentioned. However, this problem can be circumvented: The principal aspect in the present study is the creation of bremsstrahlung according to Sections 2 and 3. The creation of bremsstrahlung is always connected with scatter of impinging electrons and heat production in the related medium (usually tungsten), yet the frequency-depending dielectric factor $\varepsilon(\omega)$ appearing in all formulas of this study will be assumed to be complex, where the imaginary part describes the losses by heat production and scatter. By that, we shall make use of the dispersion methods in classical and quantum optics, where similar tasks are treated, e.g., the absorption and emission of light.

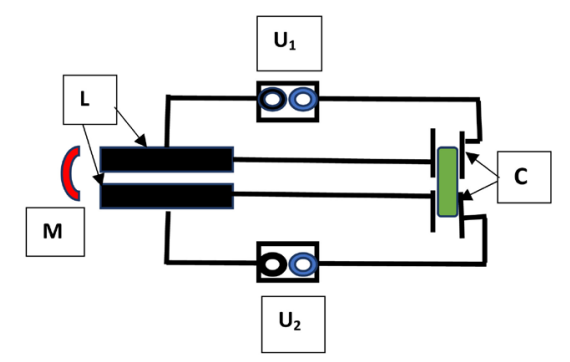

Figure 1. Two coupled circuits with mutual electric and magnetic coupling and two different driven forces. 


\subsection{Coupled Circuits with Electric and Magnetic Coupling}

In the succeeding section, it is a very essential feature that the dielectric constant $\varepsilon$ in the capacitor $\mathrm{C}$ is itself depending on the eigenfrequency $\omega_{0}$, i.e., $\omega_{0}=\omega_{0}(\varepsilon)$, in order to remove the equidistant energy levels $E_{n}=(n+1 / 2) \cdot \hbar \cdot \omega_{0}$, which are not adequate neither in low-energy molecular physics nor in radiation and highenergy physics.

This chapter is based on Figure 1 with $M \neq 0, C_{I} \neq 0$. The basic equations related to Figure 1 are:

$$
\left.\begin{array}{l}
L \cdot \ddot{Q}_{1}+M \cdot \ddot{Q}_{2}+\frac{1}{C} \cdot Q_{1}-\frac{C_{I}}{C} \cdot Q_{2}=U_{1} \cdot \mathrm{e}^{i \cdot \omega \cdot t} \\
L \cdot \ddot{Q}_{2}+M \cdot \ddot{Q}_{1}+\frac{1}{C} \cdot Q_{2}-\frac{C_{I}}{C} \cdot Q_{1}=U_{2} \cdot \mathrm{e}^{i \cdot \omega \cdot t}
\end{array}\right\}
$$

Using the substitutions $q_{1}=Q_{1}+Q_{2}, q_{2}=Q_{1}-Q_{2}, U_{11}=U_{1}+U_{2}, U_{22}=U_{1}-$ $U_{2}, \lambda_{1}=L+M, \lambda_{2}=L-M, C_{1}=C /\left(1-C_{I}\right), C_{2}=C /\left(1+C_{I}\right)$ and passing to the Lagrangean, which reads:

$$
\begin{aligned}
\not L= & \frac{\lambda_{1}}{2} \cdot \dot{q}_{1}^{2}-\frac{1}{2 \cdot C_{1}} \cdot q_{1}^{2}+U_{11} \cdot \lambda_{1} \cdot \mathrm{e}^{i \cdot \omega \cdot t} \\
& +\frac{\lambda_{2}}{2} \cdot \dot{q}_{2}^{2}-\frac{1}{2 \cdot C_{2}} \cdot q_{2}^{2}+U_{22} \cdot \lambda_{2} \cdot \mathrm{e}^{i \cdot \omega \cdot t}
\end{aligned}
$$

In Equation (3), a negative capacitive coupling is assumed, yet a positive coupling only requires the substitutions $C_{1}=C /\left(1+C_{I}\right), C_{2}=C /\left(1-C_{I}\right)$, and Equation (4) will not be changed.

The canonical momenta are given by:

$$
P_{1}=\lambda_{1} \cdot \dot{q}_{1} ; \quad P_{2}=\lambda_{2} \cdot \dot{q}_{2}
$$

By that, both Hamiltonians assume the shape:

$$
\left.\begin{array}{c}
H_{1}=\frac{1}{2 \cdot \lambda_{1}} \cdot P_{1}^{2}+\frac{\lambda_{1}}{2} \cdot \omega_{1}^{2} \cdot q_{1}^{2}-q_{1} \cdot U_{11} \cdot \mathrm{e}^{i \cdot \omega \cdot t} \\
H_{2}=\frac{1}{2 \cdot \lambda_{2}} \cdot P_{2}^{2}+\frac{\lambda_{2}}{2} \cdot \omega_{2}^{2} \cdot q_{2}^{2}-q_{2} \cdot U_{22} \cdot \mathrm{e}^{i \cdot \omega \cdot t} \\
\omega_{1}^{2}=\frac{1}{\lambda_{1} \cdot C_{1}} ; \omega_{2}^{2}=\frac{1}{\lambda_{2} \cdot C_{2}}
\end{array}\right\}
$$

The Schrödinger equation is only a slight modification of Equation (6). Therefore, we pass to the representation by creation-and annihilation operators:

$$
\left.\begin{array}{l}
P_{k} \psi \rightarrow \frac{\hbar}{i} \frac{\partial}{\partial q_{k}} \psi ; \\
\rho_{k}=U_{k} \cdot \sqrt{\frac{\hbar}{2 \cdot \lambda_{k} \cdot \omega_{k}}} ; \xi_{k}^{2}=\frac{\lambda_{k} \cdot \omega_{k}}{\hbar} \cdot q_{k}^{2} \\
\left.k=1,2 ; U_{k}=U_{11}(k=1)\right) ; U_{k}=U_{22}(k=2)
\end{array}\right\}
$$

With the help of Equation (7) the Schrödinger equation assumes the shape: 


$$
-\frac{1}{2} \frac{\partial^{2}}{\partial \xi_{k}^{2}} \psi_{k}+\frac{1}{2} \xi_{k}^{2} \psi_{k}-\rho_{k} U_{k} \psi_{k}=i \cdot \hbar \frac{\partial}{\partial t} \psi_{k}
$$

In order to introduce creation and annihilation operators we use the following relations:

$$
\left.\begin{array}{l}
b_{k}^{+}=\frac{1}{\sqrt{2}}\left(-\frac{\partial}{\partial \xi_{k}}+\xi_{k}\right) ; b_{k}=\frac{1}{\sqrt{2}}\left(\frac{\partial}{\partial \xi_{k}}+\xi_{k}\right) \\
b_{k} b_{l}^{+}-b_{l}^{+} b_{k}=\delta_{k l}(k, l=1,2)
\end{array}\right\}
$$

By that, Equation (7a) becomes:

$$
H_{k} \cdot \psi_{k}=\left[\hbar \omega_{k} \cdot\left(b_{k}^{+} b_{k}+\frac{1}{2}\right)-\rho_{k} \cdot\left(b_{k}+b_{k}^{+}\right) \mathrm{e}^{i \omega t}\right] \psi_{k}=i \hbar \frac{\partial}{\partial t} \psi_{k}
$$

\subsection{Creation of Bremsstrahlung in the MeV Domain and Application in Scanning Beam Technique of Radiotherapy and Photon-Photon Scatter (Heisenberg-Euler Scatter)}

\subsubsection{Energy Loss Determination Based on Bethe-Bloch Equation}

The creation of bremsstrahlung in a target (usually tungsten is applied) is always connected with heat production and scatter in this medium. Therefore, we have to analyze sufficiently the co-lateral processes before we are able to apply Equations (6) - (8) to this task ( $Z$ : nuclear charge of the stopping medium, $A_{N}$ : mass number, and $\rho$ : density) and have a look at Bethe-Bloch equation:

$$
\begin{aligned}
& \frac{-\mathrm{d} E(z)}{\mathrm{d} z}=\frac{K}{m c^{2} \beta^{2}} \cdot\left[\ln \left(\frac{2 m c^{2} \beta^{2}}{E_{I}\left(1-\beta^{2}\right)}\right)+a_{\text {shell }}+a_{\text {Barkas }}+a_{0} v^{2}+a_{\text {Bloch }}\right] \\
& K=\left(Z \rho / A_{N}\right) \cdot 8 \pi q^{2} e_{0}^{4} / 2 m ; \beta=\frac{v}{c} \\
& v^{2}=2 E / m ; \beta_{I}=4 / E_{I} ; E=\left(1 / \beta_{I}\right) \exp (-u / 2) \\
& \frac{1}{2} K \cdot m \cdot \beta^{2} \int \mathrm{d} z \\
& =\int \mathrm{d} u \frac{\mathrm{e}^{-u}}{u+2 \alpha_{s}+2 \alpha_{\text {Barkas }}\left(4 \cdot m / E_{I}\right)+p_{B} \mathrm{e}^{p_{B} u / 2}+\alpha_{0}\left(E_{I} / 2 m\right) \mathrm{e}^{-u / 2}}
\end{aligned}
$$

With reference to the weighted ionization potential of the stopping medium we have checked the usual value $E_{I}=75.1 \mathrm{eV}$ for water, which is tacitly assumed to be valid for other stopping media. For this purpose, we have used the ionization energy levels of the atomic shells for water, lead and tungsten determined by ICRU [7]; these energy levels have been introduced to Formulas $(9,9 \mathrm{a}, 9 \mathrm{~b})$ in order to calculate the transition probabilities $W_{i k}$ induced by collisions with external electrons. Now the average ionization potential is obtained by the weighted energy levels divided by the number of energy shells, which is $Z=10$ for water, $Z=74$ for tungsten, and $Z=82$ for lead:

$$
E_{I}=\frac{1}{Z} \cdot \sum_{i=1}^{Z} \sum_{k=1(k \neq i)}^{Z} E_{k} \cdot W_{i k}
$$


The condition $k \neq i$ must hold, since transitions can only occur by different energy levels; it should be added that Formula (9c) is a special case of the Pauli mater equation, and it provides some noteworthy results: For water, this formula yields $E_{I}=75.112 \mathrm{eV}$, which is in acceptable agreement with the assumed $E_{I}=$ $75.1 \mathrm{eV}$. The results for lead and tungsten are more interesting, since these materials are used for radiation shielding and creation of bremsstrahlung. Thus, for tungsten we obtain $E_{I}=74.45 \mathrm{eV}$ and for lead $E_{I}=84.92 \mathrm{eV}$. According to ICRU [13], the excitation energies from $1 \mathrm{~s}$ orbitals to ionization amount to 80.7556 $\mathrm{keV}$ and $79.1819 \mathrm{keV}$. The main transitions induced by electrons are between 67 $\mathrm{keV}$ and $60 \mathrm{keV}$, since the emission of radiation mainly occurs from energy levels of about $13 \mathrm{keV}$ - $15 \mathrm{keV}$ above the deepest levels, and further relevant transitions are positioned between $20 \mathrm{keV}$ and $10 \mathrm{keV}$. The relativistic corrections of the 1s-electrons of tungsten are also imposing, since a nonrelativistic calculation by the Schrödinger equation would only provide $74.45 \mathrm{keV}$. Similar results hold for lead, where the corresponding orbital excitations of 1s-electrons amount to $101.20 \mathrm{keV}$ and $99.954 \mathrm{keV}$, but the discrete emission lines are in the domain of $80 \mathrm{keV}$ and below $30 \mathrm{keV}$. The nonrelativistic determination of the 1s-electron energy now is $91.42 \mathrm{keV}$. This discrepancy results from the increase of the kinetic energy, in particular, of the innermost 1 s-electrons, by $Z^{2}$ and the relativistic mass dependence cannot be ignored.

The substitutions (9a) in Equation (9) yield the integration Formula (9b). The above Formula (9) is valid for protons and electrons, but the Barkas correction does not exist for projectile electrons, and, by setting $\alpha_{\text {Barkas }}=0$, the evaluation of the above Formula (9b) can be made easier. It is easy to verify that the correction terms prevent the singularity of the integration and by putting them to zero, the divergent integral would remain:

$$
\frac{1}{2} K \cdot m \cdot \beta^{2} \int \mathrm{d} z=\int \mathrm{d} u \frac{\mathrm{e}^{-u}}{u}
$$

We use the operator calculus developed by Feynman [14] in order to handle integration problems in quantum electrodynamics:

$$
[A+B]^{-1}=A^{-1}-A^{-2} B+A^{-3} B^{2}-A^{-4} B^{3}+\cdots+(-1)^{n} A^{-n-1} B^{n}
$$

The expression (9e) provides the integration of (9a) up to arbitrary order with finite values [15] [16] and to take account of the relativistic Bloch corrections up to arbitrary order. The divergent behavior of Equation (9), which is also reflected by Equation (9d), at low projectile energies, above all, if $E \rightarrow 0$, implies a severe aspect of Monte-Carlo calculations. The Monte-Carlo code GEANT 4 [17] uses cutoff values of the order $1-2 \mathrm{MeV}$ to guarantee reasonable results in this energy domain. The application of the procedures (9b) and (9d) circumvent the divergencies by mutual compensations of the different terms.

A practical problem in radiotherapy is to construct individual panels within an electron tube (lead allow, thickness: $1 \mathrm{~cm}$ ), since electrons create bremsstrahlung at the panel surface. 


$$
\begin{aligned}
& \left.\begin{array}{l}
R_{c s d a}=C \cdot q \cdot E_{0} \cdot\left[1-a \cdot\left(1-\mathrm{e}^{-\mu_{0} \cdot E_{0}}\right)\right] \cdot \frac{1}{f} \\
E_{0}=\frac{1}{C} \cdot a_{1} \cdot f \cdot R_{c s d a} \cdot\left(1+\varepsilon \cdot \mathrm{e}^{\mu_{1} \cdot R_{c s d a}}+a_{2} \cdot \mathrm{e}^{-\beta^{2} \cdot R_{c s d a}^{2}}\right)
\end{array}\right\} \\
& q=0.5285000251 \cdot \frac{1}{f} ; a=0.7523823 ; \mu=0.0085004 ; Z_{w}=9.75 \text {; } \\
& A_{w}=17.63 ; a_{1}=0.7570000003 \cdot f ; a_{2}=0.0128751 ; \beta=0.0035002 ; \\
& \varepsilon=0.9939000094 ; \mu_{1}=0.02639062433331674
\end{aligned}
$$

According to Formula (10) the mean stand standard deviation of $R_{c s d a}$ amounts to $0.008 \mathrm{~cm}$ and of $E_{0}$ to $0.004 \mathrm{MeV}$, if compared with ICRU, which may be downloaded by ICRU [13]. With regard to the ratio charge to mass number $\left(Z_{w}\right.$ to $\left.A_{w}\right)$ the center-of-charge and center-of-mass of $\mathrm{H}_{2} \mathrm{O}$ is more accurate. Please note that Equations $(10,10 \mathrm{a})$ refer to water with $C=1$. The correction factors $C$ and $C^{-1}$ have also to be accounted for, if one passes to high $Z$ media with different values of $E_{I}$. Thus, for tungsten we have to use $C=0.992$ and for lead $C=0.989$. A further correction refers to the factor $f$, which is $f=1$ for water (this makes sense, since water is used as the reference medium in the dosimetry), but by the fixations $Z_{m}, A_{m}$ and $\rho_{m}$ referring to the considered medium a general modification factor $f$ has to be accounted for:

$$
f=\frac{Z_{m} \cdot \rho_{m} \cdot A_{w}}{A_{m} \cdot Z_{w}}
$$

Based on Equations $(10,10 \mathrm{a}, 10 \mathrm{~b})$ the energy $E(z)$ and the stopping power $-\mathrm{d} E / \mathrm{d} z$ are given by:

$$
\left.\begin{array}{l}
E(z)=\frac{f}{C} \cdot a_{1} \cdot\left(R_{c s d a}-z\right) \cdot\left(1+\varepsilon \cdot \mathrm{e}^{\mu_{1} \cdot\left(R_{c s d a}-z\right)}+a_{2} \cdot \mathrm{e}^{-\beta^{2} \cdot\left(R_{c s d a}-z\right)^{2}}\right) \\
-\frac{\mathrm{d} E}{\mathrm{~d} z}=\frac{f}{C} \cdot\left[a_{1} \cdot\left(1+\varepsilon \cdot \mathrm{e}^{\mu\left(R_{c s d a}-z\right)}+a_{2} \cdot \mathrm{e}^{-\beta^{2} \cdot\left(R_{c s d a}-z\right)^{2}}\right)\right. \\
\left.+a_{1} \cdot\left(R_{c s d a}-z\right) \cdot\left(-\mu \varepsilon \cdot \mathrm{e}^{\mu\left(R_{c s d a}-z\right)}+a_{2} \cdot\left(-2 \beta^{2}\left(R_{c s d a}-z\right) \mathrm{e}^{-\beta^{2} \cdot\left(R_{c s d a}-z\right)^{2}}\right)\right)\right]
\end{array}\right\}
$$

A particular feature of Equation (11) is that the stopping power $(-\mathrm{d} E / \mathrm{d} z)$ remains finite at the endpoint track of the electron (Figure 2(a)):

$$
-\frac{\mathrm{d} E}{\mathrm{~d} z}=\frac{f}{C} \cdot a_{1} \cdot\left(1+\varepsilon+a_{2}\right)\left(\text { if } z=R_{c s d a}\right)
$$

With respect to the ranges of electron energies, we have restricted ourselves to $30 \mathrm{MeV}$, although we explicitly consider bremsstrahlung production of $6 \mathrm{MeV}$ and $9 \mathrm{MeV}$ electrons with tungsten targets, but this restriction is implied by two specific cases, whereas the further electron energies mainly serve to provide information on shielding problems in the radiotherapy with electrons (Figure 2(b)).

\subsubsection{Bremsstrahlung by Virtual States of the Oscillator Circuit and Applications}

Equipped with the energy deposition methods for electrons in the $\mathrm{MeV}$ domain 


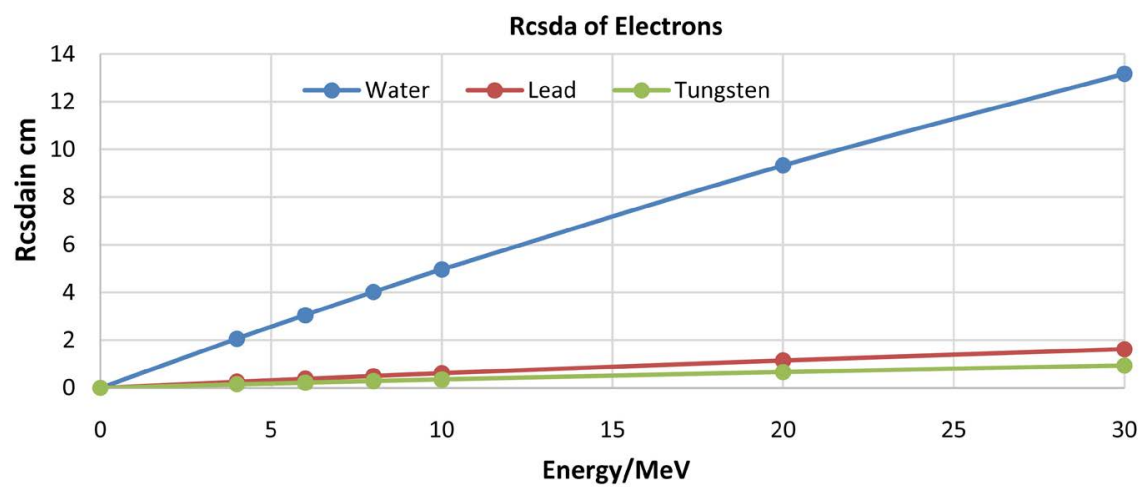

(a)

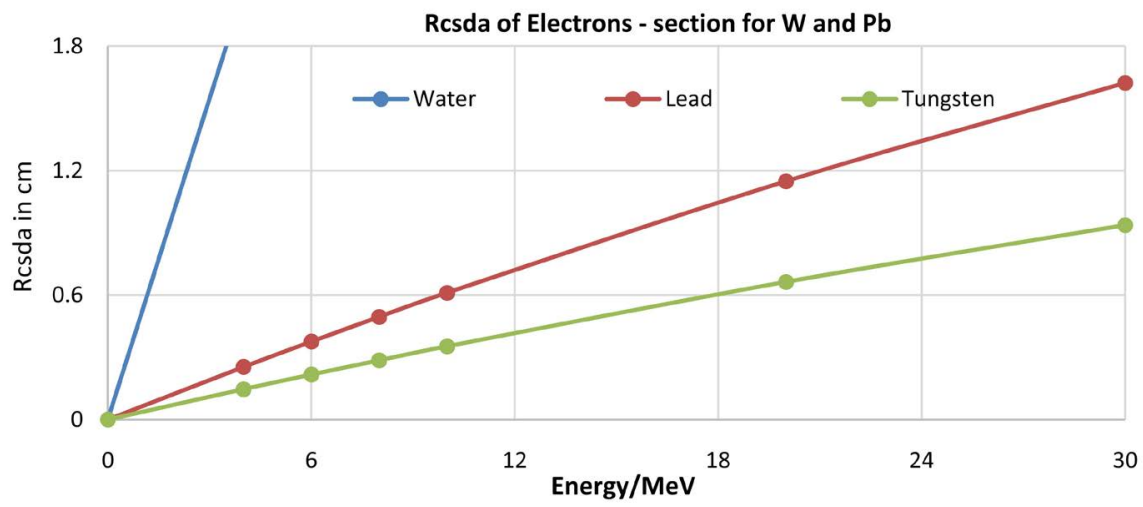

(b)

Figure 2. (a) Range of electrons depending on the initial energy $E_{0}$ and on the stopping material; (b) Section of (b) to indicate the differences between lead and tungsten.

we now pass to the bremsstrahlung creation of the differences between a conventional tungsten target and a novel target design according to Figure 3, which we reconsider with regard to a novel bremsstrahlung model [18] [19] for a new configuration of a linear accelerator (abbreviation: linac).

The thickness of each plate amounts to $0.01 \mathrm{~mm}$, and the tungsten plates are surrounded by a tungsten wall and a ferromagnet.

The distance between the plates (in vacuum) is $1 \mathrm{~mm}$, but the above construction can easier be realized, if the thickness of each plate is $0.02 \mathrm{~mm}$ and the distance between the plates $2 \mathrm{~mm}$.

Figure 4(a) completes the necessary backbone of Figure 3. The tungsten plate (red) is enveloped by a tungsten wall and a ferromagnet. With respect to the plates and the wall tungsten should be preferred, since it is paramagnetic, whereas gold is diamagnetic and weakens the magnetic field strength in the target channel. In contrast to the novel multitarget design Figure 4(b) provides the basic principle of a linac installed usually in radiotherapy stations. The incident electrons (yellow) meet the tungsten target (1 mm thick) for creation of bremsstrahlung; the succeeding plate (copper, green) primarily serve to control the target temperature, the primary collimator and the flattening filter (blue) form the profile of the outcoming radiation beam. 


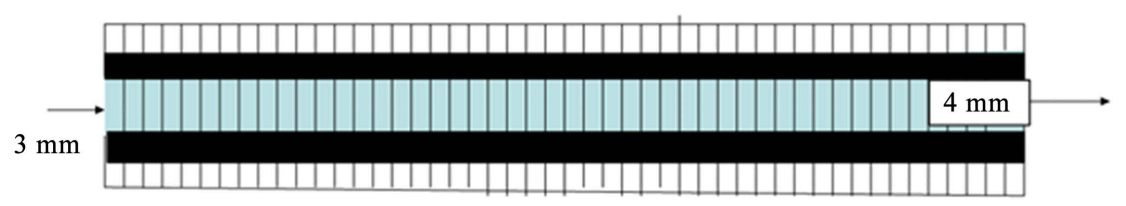

Figure 3. Cross-section of a multilayer target with 100 tungsten plates within $10 \mathrm{~cm}$ length from incident electron beam to the outgoing bremsstrahlung rays.

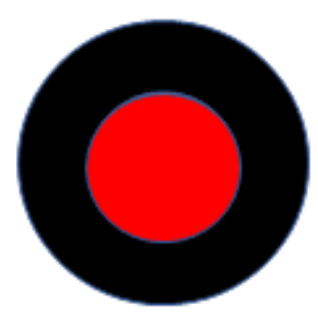

(a)

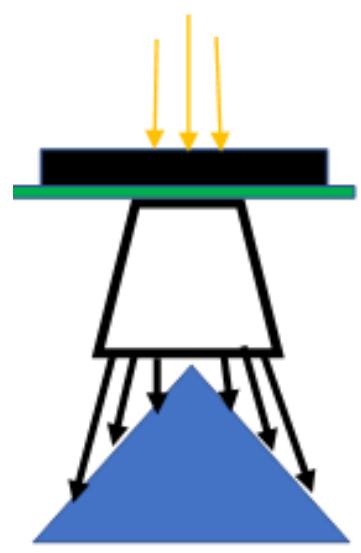

(b)

Figure 4. (a) Top view of Figure 3; (b) Schematic representation of the head of a conventional Linac.

The solution function of Equations $(7 a, 8)$ and the energy eigen-values are given by:

$$
\left.\begin{array}{l}
\psi_{k}=\sum_{n=0}^{\infty} \frac{1}{n_{k} !} \cdot\left(b_{k}^{+}\right)^{n_{k}} \cdot \mathrm{e}^{-i E_{n_{k}} t / \hbar} \cdot \mathrm{e}^{i \cdot n_{k} \cdot \omega \cdot t} \cdot \psi_{k, 0} ; \\
b_{k} \cdot \psi_{k, 0}=0 ; \quad\left(b_{k}^{+}\right)^{n_{k}} \cdot \psi_{k, 0}=\psi_{k, n} \quad(k=1,2)
\end{array}\right\}
$$

The general solution of Equation (12) is given by:

$$
\begin{gathered}
\psi_{k}=\sum_{n_{k}=0}^{\infty} \frac{1}{n_{k} !} \cdot \mathrm{e}^{-i E_{n_{k}} t / \hbar} \cdot \mathrm{e}^{i \cdot n_{k} \omega \cdot t} \cdot\left[\psi_{n_{k}}-\rho_{k} \cdot n_{k} \cdot\left(n_{k}-1\right) \cdot \psi_{n_{k}-2}\right] \\
E_{n_{k}}=\hbar \cdot \omega_{k}\left(n_{k}+\frac{1}{2}\right)+n_{k} \cdot \hbar \cdot \omega-n_{k} \cdot \rho_{k}
\end{gathered}
$$

Since the incoming electron beam has a Gaussian distribution (see also the appendix) we make use of the fixations: The field length at entrance is as usual 4 $\mathrm{mm}$, the height of the $6 \mathrm{MeV}$ Gaussian distribution at the boundaries amounts 
to $6 / e^{2}=0.135335=0.8120117 \mathrm{MeV}$. Therefore, we have to choose $e_{0} \cdot U_{1}=6$ $\mathrm{MeV}$ and $e_{0} \cdot U_{2}=0.8120117 \mathrm{MeV}$, i.e., $e_{0} \cdot U_{11}=6.8120117 \mathrm{MeV}$ and $e_{0} \cdot U_{22}=$ $5.1879883 \mathrm{MeV}$. The transition probabilities related to the yield of the bremsstrahlung spectra is received a modified version of Equation (9c) for the absorption and emission processes:

Thus, $W_{k l}$ is related to the transition probability and $P_{k}$ to the probability of the state with index $k$. The evaluation of the induced transitions with help of Formulas (12) - (14) is rather manageable by the harmonic oscillator state functions. With regard to the energy deposition of the incident electrons we have assumed further assumptions: For tungsten $(W)$ we assume $Z=74$ and $A_{N}=$ 183.840; $\mu \cdot c^{2}$ is the electron rest energy with reduced electron mass, which can be replaced by $m_{e f} c^{2}$. The ionization potentials $I_{\text {ionp,11 }}$ and $I_{\text {ionp,22 }}$ are 6.812 $\mathrm{MeV}$ and $5.188 \mathrm{MeV}$, respectively, and the ground state energy $E_{0}$ is assumed to be the electron rest energy. The density of tungsten serves as the reference value, $\delta_{\text {ref }}=19.25 \mathrm{~g} / \mathrm{cm}^{3}$. By that, the multitarget provides the ratio $\delta / \delta_{\text {ref }}=$ $0.48125 / 19.25=0.025, E_{r}$ represents the remaining electron energy, which is neither absorbed to produce dose deposition (heat) nor to create bremsstrahlung above $15 \mathrm{keV}$. The index is either $p=s$, if $U_{s}=U_{1}+U_{2}$ or $p=d$, if $U_{d}=U_{1}-U_{2}$, and $I_{\text {ionp }, p}$ as well as $E_{n, p}$ refers to the same index " $p$ " ( $I_{\text {ionp }, p}: I_{\text {ionp }, 11}$ or $I_{\text {ionp }, 22}, E_{n, p}$ : $E_{n, 11}$ or $\left.E_{n, 22}\right)$. It should also be pointed out that the zero point of $I_{\text {ionp }, p}$ has been shifted to the harmonic oscillator ground state energy, since this item remains unchanged and, therefore, Formula (14) could be slightly simplified to avoid too long formulas, in particular with regard to Formula (15).

$$
E_{n, p}=I_{i o n p, p}-\left(1-\mathrm{e}^{-\frac{n \cdot Z \cdot e_{0} \cdot U_{p} \cdot \delta}{A_{N} \cdot \mu \cdot c^{2} \cdot \delta_{r e f}}}\right) \cdot I_{i o n p, p}
$$

Therefore, the principal question is, what are the differences to the case with one single uncoupled circuit. The most interesting property of Equations (12) (14) result from the choice of $U_{1}$ and $U_{2}$, and for this purpose, we consider the creation of $6 \mathrm{MV}$ bremsstrahlung based on two different model. The use of coupled oscillators enables to simulate the energy spectrum of the incident electrons. Formula (14a) reflects the idealistic situation that the total energy of the incident electrons would be converted to bremsstrahlung.

However, Figure 5 does not correspond to the physical reality, since besides bremsstrahlung creation some additional competition effects are ignored leading mainly to heat production, but also scatter losses in spite of small angle reflection at the tungsten wall and focusing of scattered electrons by a magnetic field (field strength: 0.5 - 1.5 Tesla, in the GeV domain the field strength should be of the order 4 - 6 Tesla). The average attenuation of the photon radiation along the multitarget amounts to 0.949 , beginning with 0.99 down to 0.91 . However, the creation of radiation at the tungsten plates and the immediately attenuation is not the main contribution to heat production, since the electrons contribute to dose deposition, too. The Bragg peak of $6 \mathrm{MeV}$ electrons in tungsten is at $1.64 \mathrm{~mm}$; 


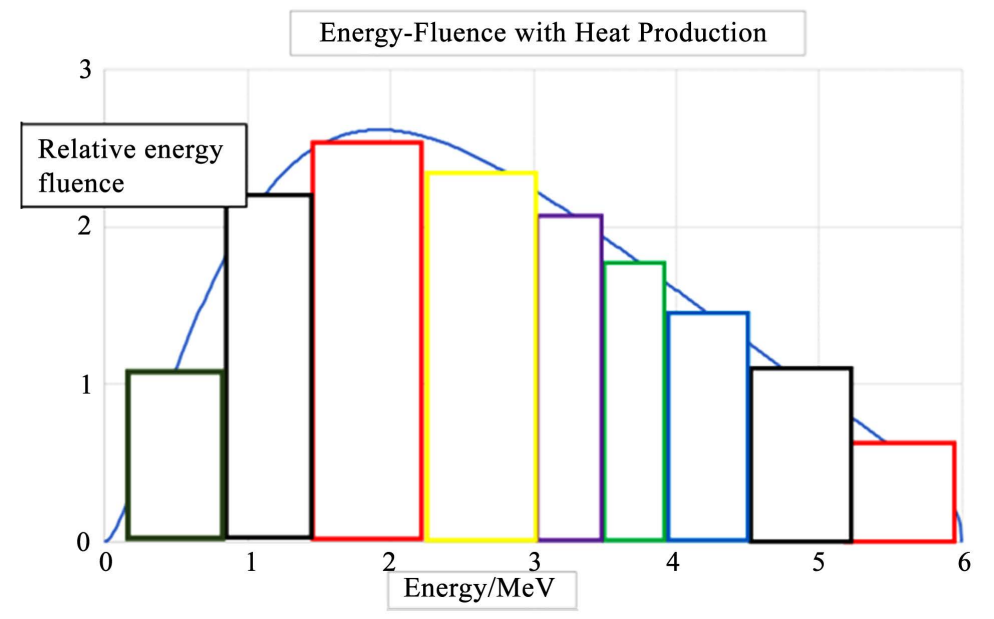

Figure 5. Calculation of the energy spectrum created by a multitarget (6 $\mathrm{MeV}$ electrons, Formula (12)).

therefore, the dose deposition in the length of $1 \mathrm{~mm}$ amounts between $25 \%$ and $40 \%$ of the incident energy. The third part of energy loss of the electrons by passing through the multitarget results from those electrons, which could not be attributed to small angle scatter and, by that, a further possibility to create bremsstrahlung is prevented. These three influences do not render to put $\lambda=1$, which would yield the creation of bremsstrahlung by the complete energy of the incident electrons, but $\lambda=0.7071$ turned out that the spectral distribution of Figure 5 is converted to Figure 6 . The contribution of the imaginary part resulting from $\sqrt{1-\lambda^{2}}$ amounts to 0.7072 .

$$
\begin{aligned}
E_{n, p}= & E_{r}+\lambda \cdot\left[I_{\text {ionp }, p}-\left(1-\mathrm{e}^{-\frac{n \cdot Z \cdot e_{0} \cdot U_{p} \cdot \delta}{A_{N} \cdot \mu \cdot c^{2} \cdot \delta_{r e f}}}\right) \cdot I_{\text {ionp }, p}\right] \\
& +\sqrt{1-\lambda^{2}} \cdot i \cdot\left[I_{\text {ionp }, p}-\left(1-\mathrm{e}^{-\frac{n \cdot Z \cdot e_{0} \cdot U_{p} \cdot \delta}{A_{N} \cdot \mu \cdot c^{2} \cdot \delta_{r e f}}}\right) \cdot I_{\text {ionp }, p}\right]
\end{aligned}
$$

With regard to the standard target according to Figure 4(a), we have to be aware of that the total length of the single tungsten target amounts to $1 \mathrm{~mm}$. Therefore, we have to put $\delta=\delta_{\text {ref }}=19.25$. The thickness for the copper plate amounts to $1 \mathrm{~mm}$, too, used to control the temperature of the tungsten target; the effective atomic charge and effective atomic number are: $Z_{\text {eff }}=76.4$ and $A_{N, \text { eff }}$ $=194.5$ and $\lambda$ has to be replaced by $0.438 \rightarrow\left(1-\lambda^{2}\right)^{1 / 2}=0.899$. By using these modifications Equation (14a) reads:

$$
\begin{aligned}
E_{n, p}= & E_{r}+0.438 \cdot\left[I_{\text {ionp }, p}-\left(1-\mathrm{e}^{-\frac{n \cdot Z_{\text {eff }} \cdot e_{0} \cdot U_{p}}{A_{N, \text { eff }} \cdot \mu \cdot c^{2}}}\right) \cdot I_{\text {ionp }, p}\right] \\
& +0.899 \cdot i \cdot\left[I_{\text {ionp }, p}-\left(1-\mathrm{e}^{-\frac{n \cdot Z_{\text {eff }} \cdot e_{0} \cdot U_{p}}{A_{N, \text { eff }} \cdot \mu \cdot c^{2}}}\right) \cdot I_{\text {ionp }, p}\right]
\end{aligned}
$$




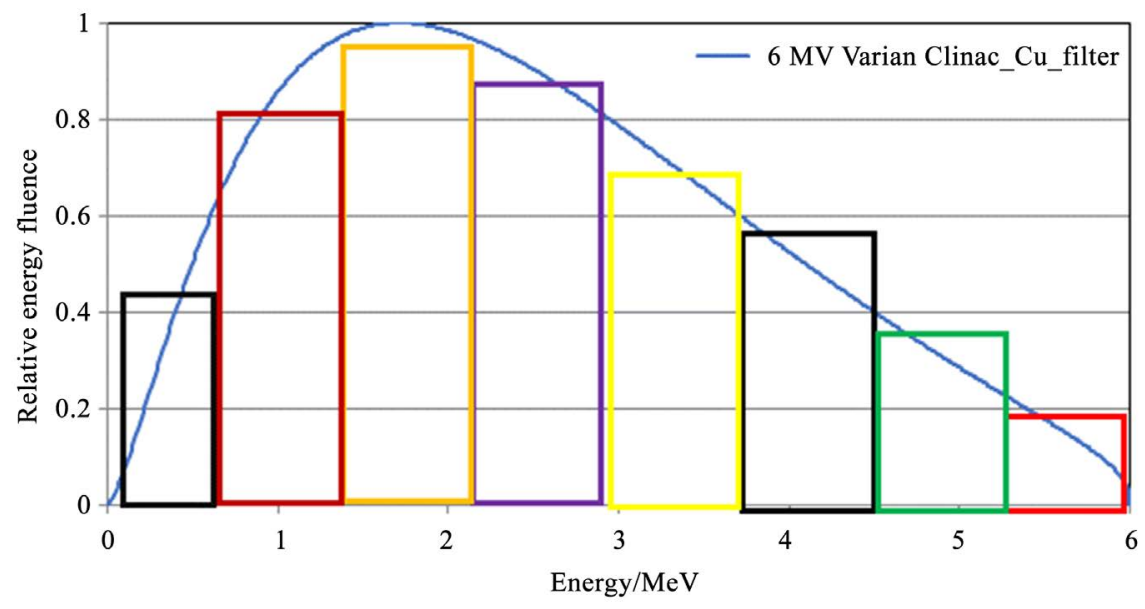

Figure 6. Calculation of the energy spectrum created by a standard target (Figure 4(a)).

Thus, $E_{n, p}$ now contains a real and an imaginary part, the latter contribution is connected to losses described above and can be associated to a heat reservoir (energy dissipation). Figure 8(b) refers to the case of $9 \mathrm{MeV}: \lambda=0.76, \rightarrow$ $\sqrt{1-\lambda^{2}}=0.65 \cdot E_{\text {average }}=4.00798418482664 \mathrm{MeV}, E_{\text {maximim }}$ at $2.98 \mathrm{MeV}$.

$$
\begin{aligned}
E_{n, p}= & E_{r}+\lambda \cdot\left[I_{\text {ion }, p}-\left(1-\mathrm{e}^{-\frac{n \cdot Z \cdot e_{0} \cdot U_{p} \cdot \delta}{A_{N} \cdot \mu \cdot c^{2} \cdot \delta_{r e f}}}\right) \cdot I_{\text {ionp }, p}\right] \\
& +\sqrt{1-\lambda^{2}} \cdot i \cdot\left[I_{\text {ionp }, p}-\left(1-\mathrm{e}^{-\frac{n \cdot Z \cdot e_{0} \cdot U_{p} \cdot \delta}{A_{N} \cdot \mu \cdot c^{2} \cdot \delta_{r e f}}}\right) \cdot I_{\text {ionp }, p}\right]
\end{aligned}
$$

The imaginary part of the above equations reflects those losses, which cannot be transmitted to bremsstrahlung. This is, above all, the production of heat in the target system and scatter. The heat production is accounted for by the function $E(z)$ according to Equation (11), whereas the influence of scatter (photons and electrons) is handled by Gaussian kernels [18] [19] [20]. The improvements of the multi-layer system with additional small angle reflection by the tungsten wall and the focusing contribution of the electrons by an appropriate magnetic field surrounding the target channel are reduction of electron scatter and heat production. The transition probabilities related to the yield of the bremsstrahlung spectra is received a modified version of Equation $(9 \mathrm{c})$ for the absorption and emission process:

$$
\dot{P}_{k}=\sum_{l=1}^{\infty}\left(W_{k l} P_{l}-P_{k} W_{l k}\right)
$$

Thus, $W_{k l}$ is related to the transition probability and $P_{k}$ to the occupation probability of the state with index $k$. The evaluation of the induced transitions with help of Formulas (12) - (15c) is rather manageable by the harmonic oscillator state functions. With $E_{0}=6 \mathrm{MeV}$ in Formula (29) only one specific incident electron energy is assumed. The parameters of Equation (16) are given in Table 1. It should be added that the Formula (16) with different parameters can also be 
Table 1. Parameters of formula (16).

\begin{tabular}{ccc}
\hline & Standard case & Multitarget focused by a magnetic lens \\
\hline $\mathrm{p}$ & 1.436 & 1.5591 \\
$\mathrm{q}$ & 0.405 & 0.4048 \\
$\beta$ & 1.055 & 1.0493 \\
$\alpha$ & 9.321 & 7.1102 \\
\hline
\end{tabular}

used for the spectral representation of other usual energies in radiotherapy, e.g., $10 \mathrm{MV}, 15 \mathrm{MV}, 18 \mathrm{MV}$, but also in the X-ray diagnostics (СТ, СВСТ, etc.). Formula (16) results from the Laplace transform of depth dose curves, providing the possibility to determine the energy spectrum, i.e., the bremsstrahlung is stated in MV instead of MeV, by experimental data received in dosimetry [20]; and this formula can be used for the calculation of the energy spectrum with the help of Table 1. However, one should take into account that its validity is restricted to an overall target of tungsten of $1 \mathrm{~mm}$, which may either be the standard or multitarget case.

$$
\left.\begin{array}{l}
f(E)=\left(1-\mathrm{e}^{-\alpha \cdot \frac{E}{E_{0}}}\right)^{p} \cdot \mathrm{e}^{-\beta \frac{E^{2}}{E_{0}^{2}}} \cdot\left(1-\frac{E}{E_{0}}\right)^{q} \\
E_{\text {average }}=\int_{0}^{E_{0}} f(E) \cdot E \cdot \mathrm{d} E / \int_{0}^{E_{0}} f(E) \cdot \mathrm{d} E
\end{array}\right\}
$$

A further restriction of the choice of the target thickness is the radiation length stated in Table 2 in order to avoid too many contributions of low-energy parts.

The average energy $E_{\text {average }}$ according to Equation (16) amounts to $2.4057 \mathrm{MeV}$ for the standard case and $2.795 \mathrm{MeV}$ for the multitarget case. In Figure 7(a), the energy fluence of the standard case is normalized to 1, but it really amounts to 0.6677 and to 1.945 for multitarget case. The drastic difference results from the absorption of the flattening filter in Figure 4(b). Due to the multitarget with the distance of $1 \mathrm{~mm}$ between each plate, we can verify two advantages, namely the backscatter at the wall and the magnetic field reduces the overall scatter and the heat production considerably. An additional copper layer for cooling the tungsten target as in Figure 4(b) is superfluous. These differences of the two configurations can be verified in Figures 5-7(a). It should be pointed out that the width of the discrete intervals has been chosen throughout in such a way, that the adaptation by the continuous function assumes the smallest difference. This is realized for all Figures in this communication such as Figure 5 and Figure 6, whereas in Figure 7(a) it appears to be inexpediently; it is appropriate with regard to Figure 8(b) and Figure 10(a) presented consecutively.

The basic physical processes occurring in the target according to the previous Figure 4(b) connected with Figure 7(b) are presented in Figure 7(c).

This Figure shows the close connection between bremsstrahlung creation and scatter (in particular, the scatter in a dense medium like tungsten). Since 


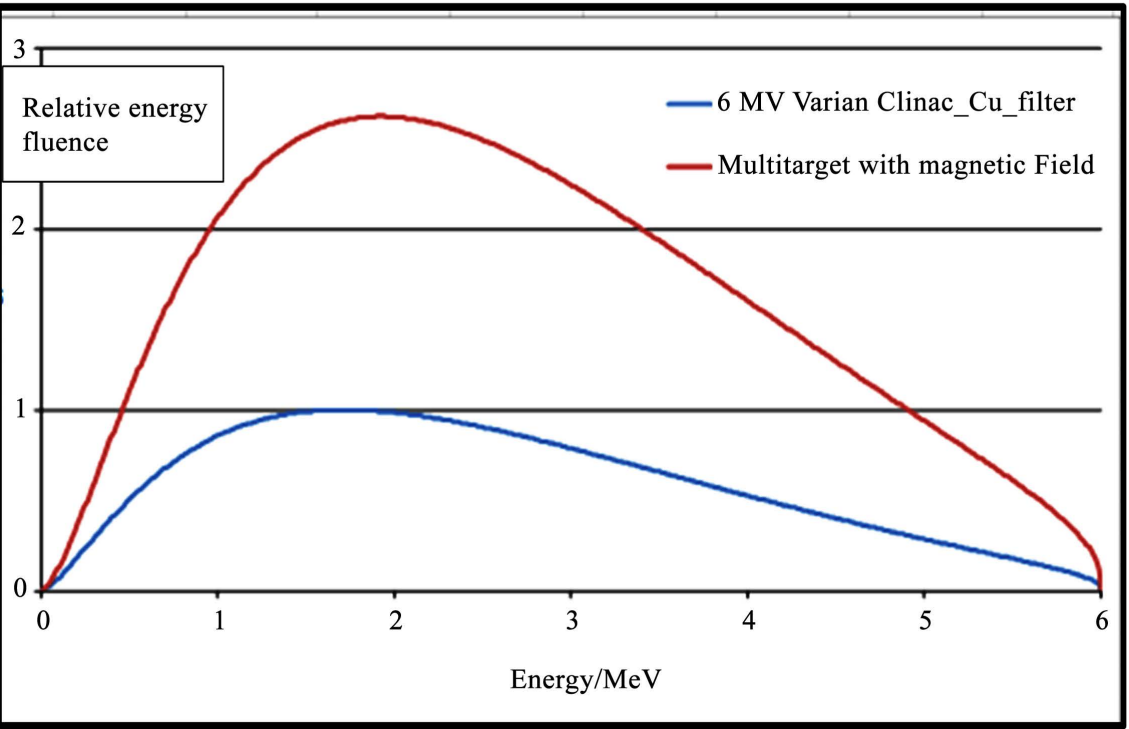

(a)

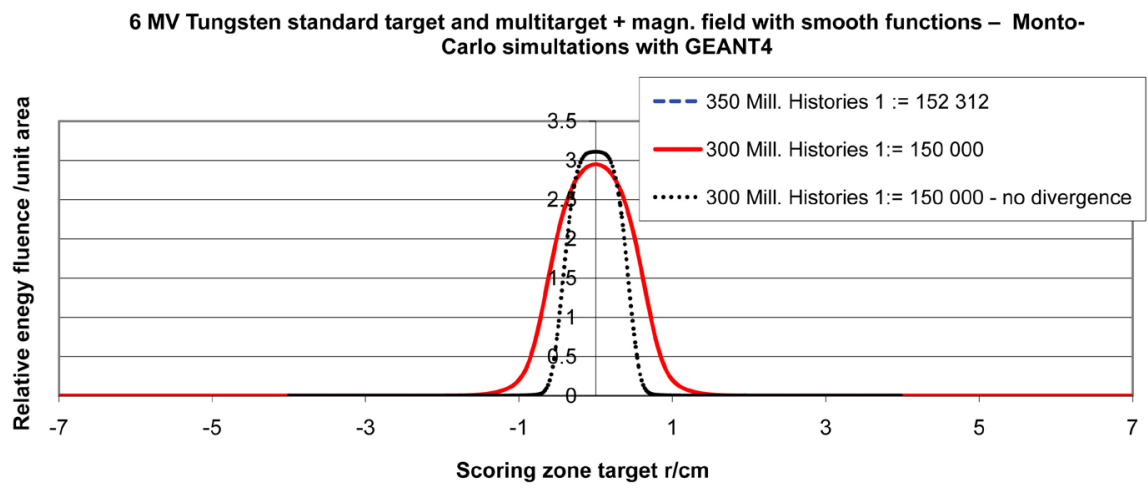

(b)

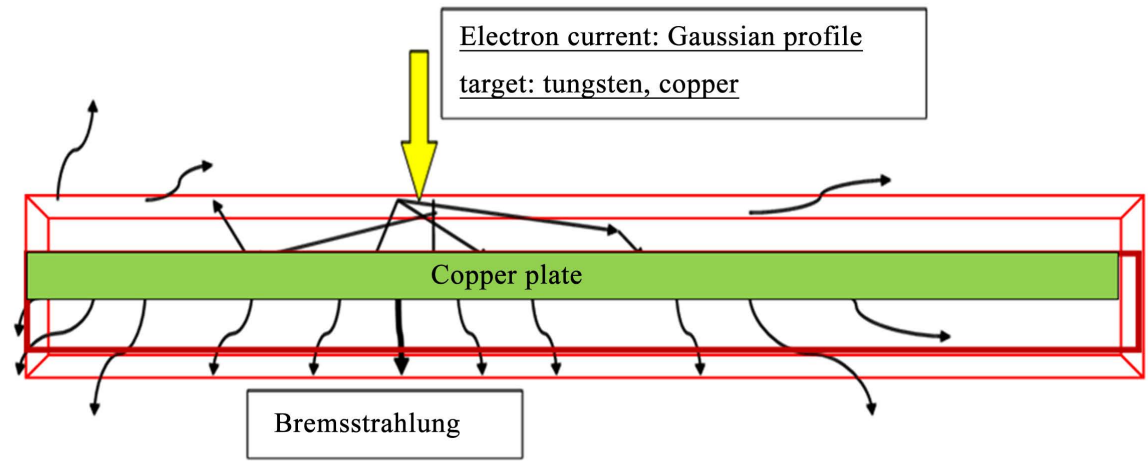

(c)

Figure 7. (a) Comparison of the energy fluences and spectra between multitarget (Figure 3 and Figure 4) and the convolution target with flattening filter (Figure 4(b)); (b) Standard target (dashes, $1 \mathrm{~mm}$ tungsten and $1 \mathrm{~mm}$ copper according to Figure 4(b), scoring domain directly below the copper layer, unit area $0.2 \mathrm{~mm}^{2}$ ). Multiple target (solid line, according to Figure 3 and Figure 4 with 100 sublayers in $10 \mathrm{~cm}$ length, field strength 0.75 Tesla, unit area $0.1 \mathrm{~mm}^{2}$ ). The conditions of the dotted curve are identical as the solid, but the field is 1.5 Tesla and the diameter at the tube end agrees with the entrance: $4 \mathrm{~mm}$; (c) Connection between creation of bremsstrahlung and scatter in a dense medium (e.g., tungsten). 

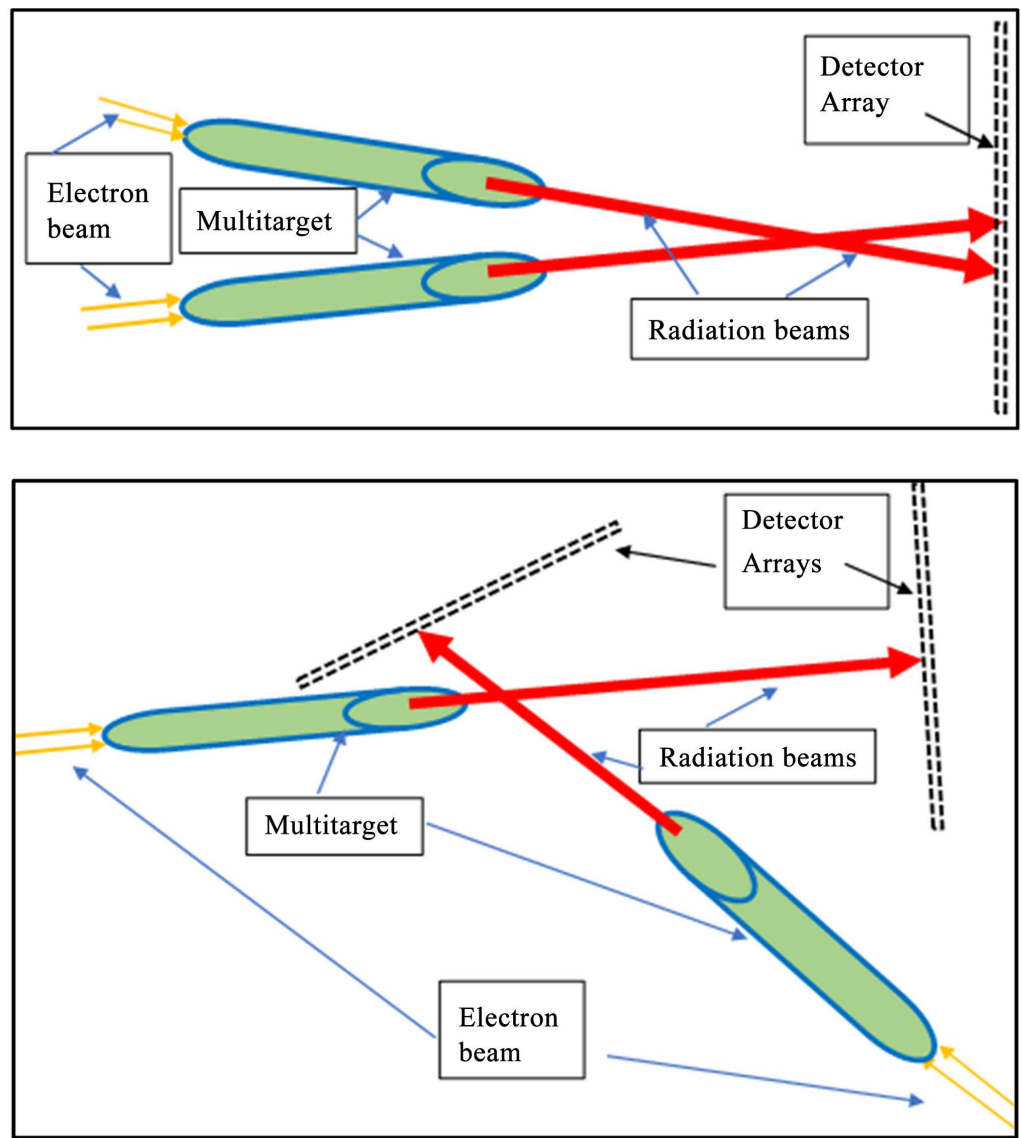

(a)

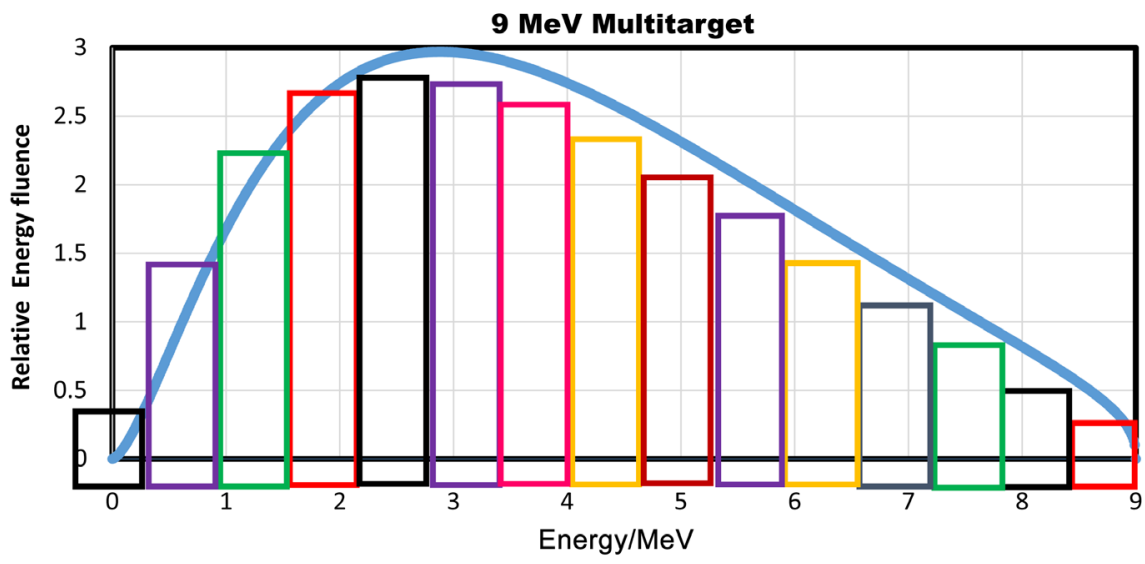

(b)

Figure 8. (a) Crossing of two narrow photon beams (energy of the photons $\geq 0.521 \mathrm{MeV}$ by two different crossing configurations to verify Heisenberg-Euler scatter of mutual photons [23]; (b) Calculation of the energy spectrum created by a multitarget $(9 \mathrm{MeV}$ electrons, Formulas (12) - (15)).

scattered electrons have to cover a longer distance, which is usually referred to as detour factor, the creation of bremsstrahlung may either be suppressed by heat production or leave the target with a large angle; this behavior is shown by the dashed curve in Figure 7(b). 
Table 2. Critical energy $E_{c}$ of bremsstrahlung creation/absorption and radiation length $\mathrm{R}_{\mathrm{l}}$.

\begin{tabular}{ccccc}
\hline Nucleus & $E_{c}$ (calculation)/MeV & $E_{c}$ (formula of thumb)/MeV & $\mathrm{R}_{1} / \mathrm{mm}$ & $\mathrm{X}_{0} / \mathrm{g} \cdot \mathrm{cm}^{-2}$ \\
\hline $\mathrm{Al}$ & 42.6230 & 42.837 & 89.924 & 24.27948 \\
$\mathrm{Fe}$ & 22.4367 & 22.3935 & 17.9550 & 14.13058 \\
$\mathrm{Cu}$ & 20.0623 & 20.1720 & 14.7577 & 13.16387 \\
$\mathrm{~Pb}$ & 8.0216 & 7.3282 & 5.5602 & 6.30527 \\
$\mathrm{~W}$ & 7.9613 & 8.1074 & 3.5151 & 6.76657 \\
$\mathrm{Au}$ & 7.5892 & 7.6022 & 3.3263 & 6.42641 \\
$\mathrm{Water}$ & 48.04 & 48.426 & 360.80 & 360.80 \\
\hline
\end{tabular}

In Figure $7(\mathrm{~b})$ the diameter of the multitarget tube at the impinging electrons amounts to $4 \mathrm{~mm}$ and at the end of the tube $6 \mathrm{~mm}$ in order to receive a connection of the solid curve with the dashed curve of the standard target according to Figure 4(b). The flattening filter is either omitted as realized in the commercially available accelerator "true-Beam" of the vendor [21] or at a different vendor [22] with regard to a scanning machine similar to a CT. Therefore, some interesting consequences result from Figures 5-7(b). A very practical aspect appears to be the construction of an ideal scanning machine in radiotherapy. In a paper [22] the bremsstrahlung of $6 \mathrm{MeV}$ is applied, and a rather small scanning beam comparable to $\mathrm{CT}$ in diagnostics performs a rotation around an iso-center. However, in a conventional technique one has to use multi-leaf collimators in order to protect some critical areas. By using the technique according to Figure 4(a) and exploiting the improved yield of bremsstrahlung the whole procedure is much more efficient, and the intensity of radiation can be steered by the electron current impinging the multi-target. Even by restriction to small-angle reflection at the tungsten wall and neglect of an additional magnetic field for focusing one receives superior results than by a target according to Figure 4(b) without flattening filter.

An interesting aspect is the creation of crossing radiation beams of photons with the energy $E_{\text {photon }} \geq$ rest energy of electrons $(0.521 \mathrm{MeV})$. In a fundamental paper dealing with photon scatter based on the Dirac equation [23], which is referred to as Heisenberg-Euler scatter, it has been pointed out that the areas of the cross-sections have to be as small as possible. In order to achieve this requirement, the multitarget system (Figure 8(a)) must not have any divergence, and the diameter of the tungsten plates is restricted to $3 \mathrm{~mm}-4 \mathrm{~mm}$. By that, an extremely high density of the photon fluence is obtained. An essential technical requirement would be a vacuum equipment for the both multiplate targets, inclusive the beam propagation to the detector array. In order to gain an optimum cross-section of the photon beams, the angle between the them should be kept as small as possible.

The configuration at the right-hand side seems to be more suitable to realize the task because of the size of the apparatuses. In both figures, we have omitted 
that the low-energy electrons leaving at the ends of the multi-target channels must be deflected by additional magnets in order to prevent disturbing effects at the detector arrays. The Heisenberg-Euler scatter is not a first-order effect in relativistic quantum theory. Figure 8(b) is the analogue to the preceding Figure 5 and Figure 6.

A significant improvement of the present configuration of Figure 3 and Figure 4 with regard to the detection of Heisenberg-Euler scatter and with regard to the $9 \mathrm{GeV}$ case in the next section, is received by the prolongation of the focusing magnetic field, which should already be active before the electron beam impinges the first subplate of the multitarget. By that, the intensity profile can be reduced to the boundary of the order $3 \mathrm{~mm}$. We should also look at the appendix, where the spectral distributions of the electrons leaving the accelerator tube are presented. Thus, we propose to omit the bypassing of the electron current by means of a bending magnet and start immediately with focusing the electron current before bremsstrahlung creation. In the case of the nominal energy $E_{0}=9$ $\mathrm{MeV}$ we also get significant contributions in the domain between 4 and $8 \mathrm{MeV}$, which certainly enhances the measurement conditions.

The magnetic field strength leading to Figure 8 (b) has assumed to be 1.5 Tesla, if the channel diameter amounts to $4 \mathrm{~mm}$. If this diameter is reduced to 3 $\mathrm{mm}$, the magnetic field strength has to be increased, but 2 Tesla would be sufficient.

\subsubsection{Application in the keV Domain: Scanning Method with a Multitarget Beam}

A look on Formula (14a) shows that for energies in the keV domain, where the voltage is of the order of about $120 \mathrm{kV}, e_{0} \cdot U_{p}$ is much smaller than the electron rest energy $\mu c^{2} \cong m_{\text {electron }} \cdot c^{2}$. Therefore, the essential term in the exponential function is only of first order and terms of higher order than $\mathrm{Z}^{1}$ only provide small corrections (this aspect is also analyzed in Section 3.1). This fact provides an interesting proposal with regard to the CT-scanning methods: An attractive version of the cited scanning machine in the $\mathrm{MeV}$ domain [24] would be the analogue design in the CT domain. The wall should also consist of tungsten and an additional magnetic field for focusing should be applied. However, the effective overall density of tungsten must be reduced, and only about 10 plates with $0.01 \mathrm{~mm}$ thickness of each plate could be considered (the overall thickness amounts to $0.1 \mathrm{~mm}$ ), the distance between the plates must also be increased and should amount to $5 \mathrm{~mm}$. The diameter of the multilayer channel according to Figure 3 can amount to $3-4 \mathrm{~mm}$ and may depend on further technical requirements of the construction. Such a construction of a multitarget for CT applications would consume a total length of about $5 \mathrm{~cm}$, and some benefits may be pointed out: The exploitation of bremsstrahlung via backscatter is removed, heat production is essentially reduced and cooling is superfluous. The profiles are rigorously symmetric - an additional wedge filter to remove the asymmetry is not required. It should also be mentioned, that the effective overall density of 
tungsten can be reduced by the application of sublayers of which the thickness amounts to $0.005 \mathrm{~mm}$. Then 20 subplates with identical distance of $5 \mathrm{~mm}$ can lead to a total length of $10 \mathrm{~cm}$, and the focusing effect is increased. The main benefit of this proposal is, besides sparing electric energy of the supplying system, the scatter of the bremsstrahlung within the scanning beam is reduced, which implies also an improvement of the image construction. Since the energy of the impinging electrons is restricted to $100 \mathrm{keV}-140 \mathrm{keV}$, the field strength of the focusing magnetic field can be reduced compared to the $\mathrm{MeV}$ domain, and 0.5 Tesla - 1 Tesla are sufficient.

In Figure 9 the bremsstrahlung created by the standard target (solid curve) is normalized to " 1 " at the energy $E=39.95 \mathrm{keV}$. The related average energy $E_{a v}$ amounts to $E_{a v}=57.68 \mathrm{keV}$. The dashed curve is the result of the multitarget described above with 10 layers (thickness $0.01 \mathrm{~mm}$, distance between 2 layers: 5 $\mathrm{mm})$. The magnetic field strength is assumed to be 0.75 Tesla. The maximum of the bremsstrahlung amounts to $E=44.67 \mathrm{keV}$ and the related average energy to $E_{a v}=64.67 \mathrm{keV}$. The characteristic peaks of tungsten resulting from shell transitions can be verified in both cases. It has to be mentioned that discrete interval steps would only be possible in the dashed curve referring to the multitarget with a diameter of $4 \mathrm{~mm}$, whereas the solid curve results from a different technique, namely the reflection at a rotating tungsten disk. The scoring plane is immediately the disk. If one traces the produced beam up to the jaws, a divergent broad beam can be recognized, since the impinging electron beam at the tungsten disk is oblige. Therefore, a discrete representation of relative energy fluence does not make sense due to the lack of comparability.

\subsection{Extension to the $\mathrm{GeV}$ Domain with $9 \mathrm{GeV}$ as an Example}

The photon-photon scatter originally considered by Heisenberg \& Euler seems to an indication that with very narrow and, by that, high intensity beams should

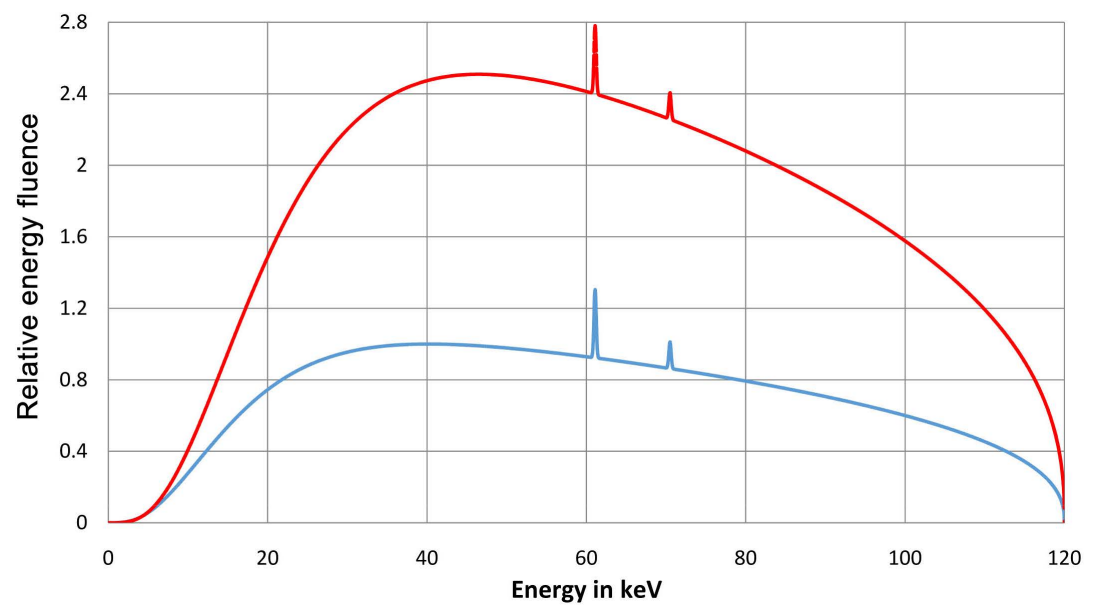

Figure 9. Comparison of the energy fluence of $120 \mathrm{keV}$ between standard target resulting from the conventional backscatter method with the help of Monte-Carlo simulations and the focused multitarget. 
be extended to the $\mathrm{GeV}$ domain. However, this task may imply some performance difficulties, namely to create two crossing beams in this energy domain. Therefore, the main motivation to make use of modifications of a standard target according to Figure 3 and Figure 4 is the yield of GeV-bremsstrahlung in photon-hadron interactions [24] in order to receive further insights in high energy physics besides proton-proton collisions (e.g., the LCH of CERN). The drawing 9 gives some essential information on the present conception, which is based on $9 \mathrm{GeV}$ electrons. As in the preceding case according to Figure 3 and Figure 4 we assume 100 subplates of tungsten with thickness of each amounts to $0.01 \mathrm{~mm}$, i.e., the overall thickness is $1 \mathrm{~mm}$. The distance between two subplates should, at least, amount to $1 \mathrm{~mm}$, but the use of 2 or more millimeters may improve the results, since the average density of the tungsten multitarget is reduced. The complete configuration is, as previously considered, based on small angle scatter at the tungsten wall with the following meaning: In Figure 9 we denoted by the case 1 typical small angle scatter. From a physical viewpoint we have to be aware of multiple Coulomb scattering, but in this situation there a more electrons in the (dense) medium tungsten, which reject the penetrated electron, since the repulsive force is predominating. The case 2 shows an electron with a somewhat increased scatter angle, where the final behavior can only be predicted by the probability to leave the wall like the case 1 or the electron may take a longer path within the wall, before it may leave it again (case 3) with significant loss of energy. Since the radiation length of tungsten amounts to 3.5 $\mathrm{mm}$ (Table 2), the wall should approximately incorporate a comparable thickness.

Keeping the physical background of Figure 10(a) in mind we should like to mention a severe problem of bremsstrahlung creation in ultrahigh energy physics, which is referred to as LPM-effect (Landau, Pomeranchuk, Migdal), according to this effect in high dense media such as tungsten the multiple Coulomb scattering [25] suppresses the creation of bremsstrahlung [26] [27], and the original electron energy either goes lost by heat production or the bremsstrahlung with significantly lower energy leaves the target with a large angle to be useless. Figure 7(b) and Figure 10(a) point out the close connection between scatter and creation of [17]. The multilayer conception with small angle scatter at the wall exploits the LPM effect, since under this condition the repulsion of the electrons in wall material is dominant and the electron is rejected by a rather high probability. On the other hand, the very thin plates imply that either the LPM effect is quenched or, at least, reduced. The studies of the authors mentioned above are fixed to a single target. Therefore, the computational studies with GEANT 4 and experiments have been restricted to a target with thickness 0.05 multiplied with radiation length. With regard to tungsten this restriction implies a target thickness of $0.17 \mathrm{~mm}$ [26] [27], i.e., in the present study 17 subplates would be considered. This situation is a clear indication that the yield of bremsstrahlung creation should be improved and forced up. The result of the 


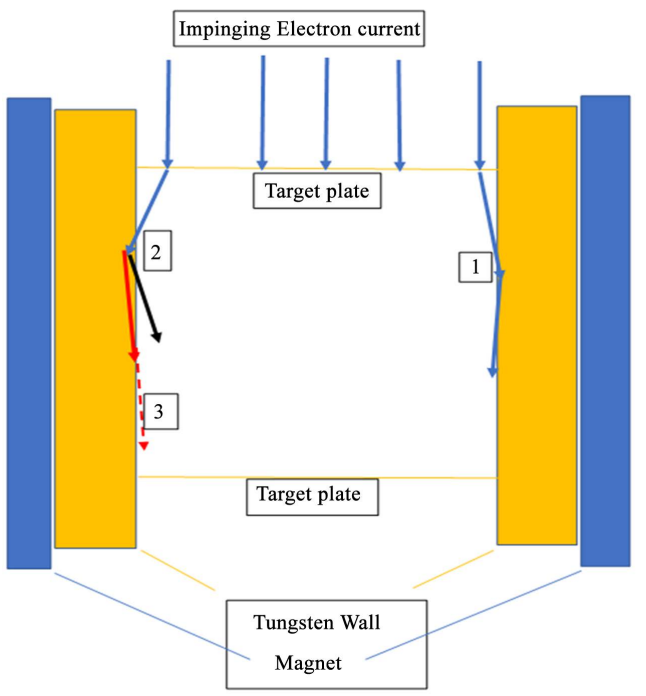

(a)

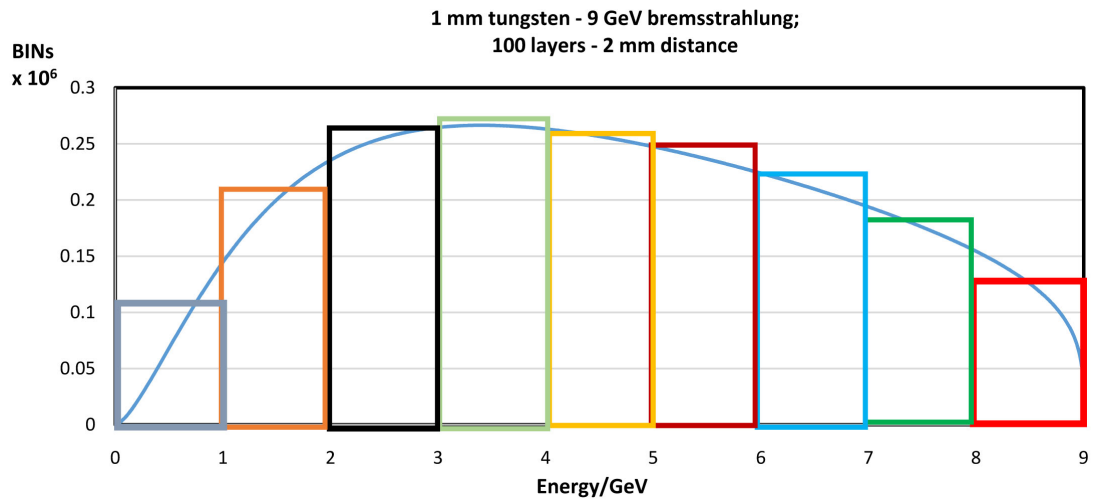

(b)

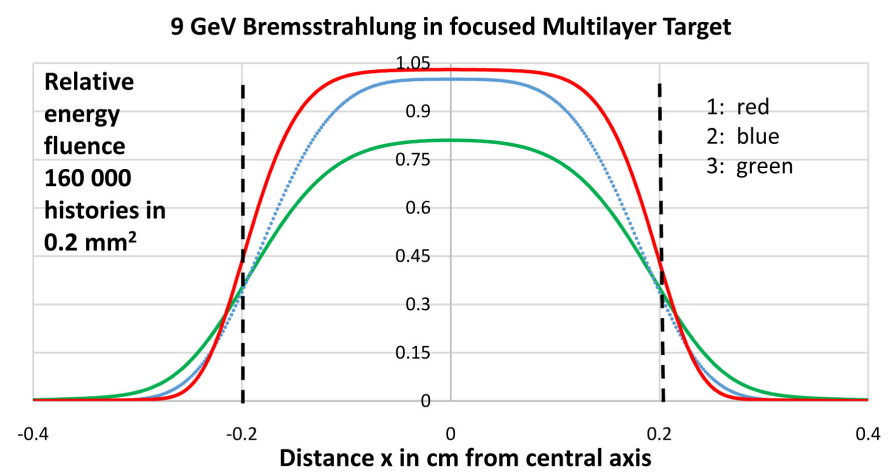

(c)

Figure 10. (a) Role of small angle scatter at a tungsten wall and additional support of a focusing magnet; (b) Creation of bremsstrahlung based on a multi-target useful in high energy physics; (c) A common feature of the three curves is that the multitarget tube is free of divergence, the diameter amounts to $4 \mathrm{~mm}$ and 100 tungsten sublayers with a total thickness of $1 \mathrm{~mm}$ is assumed, i.e., the thickness of each sublayer amounts to $0.01 \mathrm{~mm}$. The distance between the sublayers is $1 \mathrm{~mm}$ in the case 2 and 3 (case 2 serves as the normalization), but in case 3 the field strength is 3 Tesla (solid), in case 25 Tesla (dots). Case 1 (dashes) differs from case 2 by the total multilayer tube length, which is no $20 \mathrm{~cm}$ (5 Tesla, distance between the layers: $2 \mathrm{~mm}$ ). 
present multitarget configuration with tungsten wall and magnetic field can be verified in Figure 9(a). With regard to the additional magnetic field to reach an improvement and exploitation of bremsstrahlung we refer to previous publications [18] [19]. The principal difference to the mentioned publications is a significantly modified and improved theoretical method, based on coupled circuits with inclusion of polarization effects of high energy electrons. Thus, Figure 9(a) presents two different procedures, namely the collection of BINs at the subplates and the continuous envelope function according to Equation (16).

The spectral distribution related to Figure 10 (b) is determined by Equation (15). The principal difference is the numerical value of $\lambda$, which is now $\lambda=$ 0.8096 , and, related to the imaginary part, the numerical value of $\left(1-\lambda^{2}\right)^{1 / 2}=$ 0.586982 .

As already pointed out in the previous section (Heisenberg-Euler scatter), the basis of Figure 10(c) can be significantly improved by a prolongation of the focusing magnet before entering the multitarget in order to reach a "beam-diameter" of $3 \mathrm{~mm}$ instead of the $4 \mathrm{~mm}$ in Figure 10(c).

\section{Discussion}

The problem of the bremsstrahlung creation concentrated to small field sizes with relevance to novel irradiation techniques has already studied in ref. [28], which might be regarded as the precursor of the present analysis. In this study, the authors considered a sequence of Beryllium (Be) targets instead of tungsten, since the low atomic charge number of Be with $Z=4$ yields reduced scatter of the impinging electrons. However, this lower charge number with $Z=4$ requires electron energies of about $60 \mathrm{MeV}$ in order to produce a bremsstrahlung energy spectrum comparable with $6 \mathrm{MeV}$ by using a tungsten target, and the electrons with remaining energies $E<40 \mathrm{MeV}$ had to be deflected by a magnetic field below the Be targets. Some essential features of the present study, namely reflection of small angle scattered electrons at the tungsten wall and the focusing influence of a surrounding magnetic field, have not been accounted for in [28]. If we have a look to the Formula (14a) and the subsequent formulas in section 2.2, we can readily verify that a low $Z$, e.g., $Z=4$ at Be, requires a corresponding increase of the impinging electron energy with respect to bremsstrahlung creation. However, the relationship between input energy of the electrons and the yield of bremsstrahlung is not impressive, and, by that, the proposal [28] was not realized.

\subsection{Properties of the Exponential Bremsstrahlung Spectra and the Role of the Heat Production}

Section 2 shows that coupled circuits can find applications in different energy regions of physics. Thus, in various physical disciplines the harmonic oscillator approach with constant differences between the energy levels may not be suitable to describe actual problems. There are two different ways to overcome this 
problem: 1) One may use harmonic oscillator conformations and take account for the polarizability of the dielectric constant in dependence of the energy under consideration. This behavior is increasing with increasing energy. By that, the whole task turns out to be a nonlinear one. 2) A further access to overcome this problem uses nonlinear field theory either in position space [3] or in charge space [4]. In both field-theoretical ways, there exists the possibility to transform the nonlinear and nonlocal field equations to self-interacting oscillators, where the eigen-frequencies depend on the wave-function themselves. Then a low-order approach valid only to a few excitations is a harmonic oscillator with self-interaction.

The essential term of Formulas (13) - (15) is related to the exponential energy spectrum creation of bremsstrahlung resulting from mutual couplings ( $M$ and $C_{I}$ ) of the circuits according to Figure 1 and of energy dependence of the polarization at the capacitors $\varepsilon(\omega)$, which have to be chosen such, that the energy difference between virtual state $n=1$ and the upper limit $n \rightarrow \infty$ matches the boundary condition of the forced oscillator $e_{0} \cdot U_{p}-E_{a v}$ (average energy of the target material, which is for tungsten $E_{a v}=74.45 \mathrm{eV}$, see Section 2). For the following discussion the basic equation (14a) is repeated here:

$$
E_{n, p}=I_{\text {ionp }, p}-\left(1-\mathrm{e}^{-\frac{n \cdot Z \cdot e_{0} \cdot U_{p} \cdot \delta}{A_{N} \cdot \mu \cdot c^{2} \cdot \delta_{r e f}}}\right) \cdot I_{\text {ionp }, p}
$$

The power expansion of the parenthesis of this formula can be used to provide further results:

$$
-\left(1-\mathrm{e}^{-\frac{n \cdot Z \cdot e_{0} \cdot U_{p} \cdot \delta}{A_{N} \cdot \mu \cdot c^{2} \cdot \delta_{r e f}}}\right)=-\left[1+\frac{n \cdot Z \cdot e_{0} \cdot U_{p} \cdot \delta}{A_{N} \cdot \mu \cdot c^{2} \cdot \delta_{r e f}}-\frac{1}{2}\left(\frac{n \cdot Z \cdot e_{0} \cdot U_{p} \cdot \delta}{A_{N} \cdot \mu \cdot c^{2} \cdot \delta_{r e f}}\right)^{2}+\cdots\right]
$$

The connection of the expansion (17a) with Formula (17) shows that for energies in the $\mathrm{keV}$ domain, where the voltage is of the order $100 \mathrm{kV}, e_{0} \cdot U_{p}$ is much smaller than the electron rest energy $\mu c^{2} \cong m_{\text {electron }} \cdot c^{2}$. This property has been used in Section 2.2.3, in which we have described an application to CT methods. Therefore, the terms of higher order than $Z$ only provide extremely small corrections.

On the other hand, the higher order terms $Z^{2}, Z 3, \ldots$, become significant with increasing electron energy in the $\mathrm{MeV}$ domain. By that, we can conclude that the developed formalism works in the $\mathrm{keV}$ as well as in the $\mathrm{MeV}$ domain, and the main effort is the evaluation of the transition probabilities. In the following Section 5 we present some indication that the elaborated methods also work in the $\mathrm{GeV}$-domain, since the exponential expression already includes all terms of higher order, and the multitarget according to Figure 3 improves the yield of bremsstrahlung. We have already pointed out that for electron energies below 1 $\mathrm{GeV}$ the heat production due to multiple Coulomb scattering cannot be neglected. Therefore, we have checked the previous formulas based on the integration of the relativistic Bethe-Bloch equation can readily be extended to energies 
of the order of $1 \mathrm{GeV}$.

$$
\left.\begin{array}{l}
R_{c s d a}=\left[\sum_{k=1}^{4} a_{k} \cdot E_{0}^{k}+a_{5} \cdot E_{0}^{p}\right] \cdot \frac{C}{f} \\
a_{1}=0.4092031 ; a_{2}=-9.80895 \times 10^{-4} ; a_{3}=1.16056 \times 10^{-6} ; \\
a_{4}=-4.95957 \times 10^{-10} ; a_{5}=0.67168125 ; p=9.0875 \times 10^{-2} \\
E_{0}=b_{1} \cdot R_{c s d a} \cdot \mathrm{e}^{\mu \cdot R_{c s d a}}\left[1-b_{2} \cdot R_{c s d a}^{q}\right] \cdot \frac{C}{f} \\
b_{1}=13.1503746738472 ; b_{2}=0.787625044162269 ; \\
\mu=2.331100073934067 \times 10^{-2} ; q=3.527749515160394 \times 10^{-2}
\end{array}\right\}
$$

The present calculation formulas for the Rcsda range and related energy as the inverse task represent slight modifications of the previous formulas valid up 30 $\mathrm{MeV}$, valid for the energy range $>30 \mathrm{MeV}$. If compared with ICRU [7], the average standard deviation for Rcsda amounts to $0.95 \mathrm{~cm}$, and with regard to the inverse task this deviation amounts to $0.51 \mathrm{MeV}$. The determination of the residual energy $E(z)$ agrees with Formula (18), since only the substitution $R_{c s d a} \rightarrow$ $R_{c s d a}-z$ has to be performed. However, we should finally point out that only Formula (10) - (11) are recommended for the energy domain of interest in the radiotherapy with electrons.

\subsection{Some Aspects of the Voltages of Coupled Oscillators}

A noteworthy feature emerges by the role of the voltages, which may either be time-dependent $U_{1} \cdot \mathrm{e}^{i \omega t}$ and $U_{2} \cdot \mathrm{e}^{i \omega t}$ or static by setting $\omega=0$. Due to the mutual coupling of the circuits according to Figure 1 the voltages of the normal modes appear as sums of the two voltages as well as the differences, i.e., $U_{11}=U_{1}+U_{2}$ and $U_{22}=U_{1}-U_{2}$. In the present study we have fixed: $U_{1}=6 \mathrm{MeV}, U_{2}=0.812$ $\mathrm{MeV} ; U_{1}=9 \mathrm{MeV}, U_{2}=0.95 \mathrm{MeV} ; U_{1}=9 \mathrm{GeV}, U_{2}=0.7 \mathrm{GeV}$. By that, we obtain: $U_{11}=6.812 \mathrm{MeV}, U_{22}=5.188 \mathrm{MeV} ; U_{11}=10.218 \mathrm{MeV}, U_{22}=7.782 \mathrm{MeV}$; $U_{11}=9.7 \mathrm{GeV}, U_{22}=8.3 \mathrm{GeV}$ (in the latter case the numerical values are estimated, since the Gaussian distribution may probably be invalid). These assumptions may adapt the Gaussian distributions of the incident electron current. Since due to the mutual couplings only the sums and differences of $U_{1}$ and $U_{2}$ enter the calculation procedures of the energy spectra, we receive much higher flexibility, e.g., by putting $U_{1}=U_{2}$, the sum $U_{11}=2 \cdot U_{1}$, whereas $U_{22}$ assumes 0 . This means that one normal mode is not coupled to a force!

\subsection{A Practical Aspect: The Shielding Problem in Radiotherapy with MeV Electrons}

A further aspect of the received results is a side-effect, the shielding problem with respect to individual cover plates in radiotherapy of electrons. Thus, the above Formula (10a) can be used for the calculation of the energy loss of a lead alloy. On the other hand, the creation of bremsstrahlung in this alloy is a severe problem, insofar the individual cover plate does not exhibit the sufficient thickness. If one uses $4 \mathrm{MeV}$ or $6 \mathrm{MeV}$ electrons suitable for subcutaneous irradiation 
a shielding thickness of $2 \mathrm{~cm}$ would be acceptable, but the application of electron energies beyond $6 \mathrm{MeV}$ must account for increasing thickness of the shielding alloy else the undesired irradiation with bremsstrahlung produced in the shielding material would occur.

\section{Conclusions}

The quantum theoretical treatment of circuits implies similar as in the quantum mechanical case equidistant energy levels, if the material properties of the capacitor do not depend on the energy domain. This assumption is, in general, not valid, and the relation $C=\varepsilon \cdot C_{0}$ has to be replaced by $C=\varepsilon(\omega) \cdot C_{0}$, i.e., the dielectric constant $\varepsilon$ depends on the frequency of energy due to the polarization interaction. Thus, if electrons interact with numerous electrons in a material, polarization effects will always consequently be evoked. This behavior is not only restricted to existing electrons, e.g., in a metal like tungsten, but also important for fast electrons traveling in vacuum by inducing the so-called "vacuum polarization" by interaction with virtual electrons and positrons [14].

In this study, we have made use of the polarization effects in the capacitors in order to account for the induced virtual states necessary at the creation of bremsstrahlung. An improvement and refinement of the presented conception would be the use of three (or more) mutually coupled circuits. However, it appears that in the $\mathrm{MeV}$ domain with predominance in radiotherapy this extension is not required. Therefore, only the bremsstrahlung production in the high energy physics could be described by a refined conception, in particular, if one passes to electron energies beyond the domain of $9 \mathrm{GeV}$. Since the particular view of this study are quantized circuits, the results are restricted to distributions in the energy space, and the spatial distributions are ignored. However, this shortcoming has been removed by further information resulting from Monte-Carlo calculations with the code GEANT 4. It should also be added that it is certainly attractive to study the bremsstrahlung production besides $9 \mathrm{GeV}$ of further ultra-high energies in order to elucidate quark and gluon properties by a deeper level, since the LPM-effect can be reduced drastically by the wall reflection in a multitarget system and the focusing influence of magnetic fields. Since uranium, which is also paramagnetic, might possibly more suitable for these studies, a substitution of tungsten by uranium could provide better results. The theoretical procedure developed in Section 2 has not to be extended.

The introductory part has provided information about the wide field of quantized circuit models, such as quantum optics, Josephson junctions in superconductivity, molecular biophysics and physical basis of biorhythms. It appears that this field of quantized circuits may be completed by additional references [29]-[34] with regard to the mentioned topics, which provide a clear indication that these novel research fields can lead to new application fields in physics and technical problems. Therefore, the present study may serve as a contribution of an attractive extension of new possible applications of quantum theory. 


\section{Conflicts of Interest}

The author declares no conflicts of interest regarding the publication of this paper.

\section{References}

[1] Ulmer, W. (2015) Quantum Theory of Friction and Electric Resistance of Circuits and Applications to Radiation Physics. International Journal of Innovation in Science and Mathematics, 3, 2347-9051.

[2] Ulmer, W. (2015) Quantum Theory of Coupled Electromagnetic Circuits-Extensions and Transitions to the Continuum and Applications to Problems with Spin and Nuclear Physics. International Journal of Innovation in Science and Mathematics, 4 , 302-314.

[3] Ulmer, W. (1980) On the Representation of Atoms and Molecules as Self-Interacting Field with Internal Structure. Theoretica Chimica Acta, 55, 179-205. https://doi.org/10.1007/BF00556156

[4] Ulmer, W. (2019) Quantum Theory of Circuit Systems-I. Aspects of Symmetry Principles and a Generalization of the Nonlinear Schrödinger Equation in Charge Space. Journal of Nuclear and Particle Physics, 9, 59-68.

[5] Ulmer, W. and Cornélissen, G. (2013) Coupled Electromagnetic Circuits and Their Connection to Quantum-Mechanical Resonance Interactions and Biorhythms. Open Journal of Biophysics, 3, 253-274. https://doi.org/10.4236/ojbiphy.2013.34031

[6] Chen, W.Y. (2004) Home Networking Basics. Prentice Hall, Hoboken.

[7] Van Zon, R., Ciliberto, S. and Cohen, E. (2004) Power and Heat Fluctuation Theorems for Electric Circuits. Physical Review Letters, 92, Article ID: 130601. https://doi.org/10.1103/PhysRevLett.92.130601

[8] Devoret, M.H. and Martinis, J.M. (2004) Implementing Qubits with Superconducting Integrated Circuits. Quantum Information Processing, 3, 163-203. https://doi.org/10.1007/s11128-004-3101-5

[9] Schuch, D., Chung, K.M. and Hartmann, H. (1983) Non-Linear Schrödinger-Type Field Equation for the Description of Dissipative Systems. I. Derivation of the Nonlinear Field Equation and One-Dimensional Example. Journal of Mathematical Physics, 24, 1652-1660. https://doi.org/10.1063/1.525861

[10] Schuch, D., Chung, K.M. and Hartmann, H. (1984) Nonlinear Schrödinger-Type Field Equation for the Description of Dissipative Systems. III. Frictionally Damped Free Motion as an Example for an Aperiodic Motion. Journal of Mathematical Physics, 25, 3086-3092. https://doi.org/10.1063/1.526024

[11] Tsekov, R. (2012) Nonlinear Friction in Quantum Mechanics. Ann. Univ. Sofia, Faculty Physics, 105, 14-21.

[12] Tsekov, R. and Ruckenstein, E. (1994) Stochastic Dynamics of a Subsystem Interacting with a Solid Body with Application to Diffusive Processes in Solids. The Journal of Chemical Physics, 100, 1450-1454. https://doi.org/10.1063/1.466623

[13] Berger, M.J., Coursey, J.S. and Zucker, M.A. (2000) ESTAR, PSTAR and ASTAR: Computer Programs for Calculating Stopping-Power and Range Tables for Electrons, Protons and $\alpha$-Particles (Version 1.2.2). National Institute of Standards and Technology, Gaithersburg.

[14] Feynman, R.P. (1962) Quantum Electrodynamics. W.A. Benjamin, New York.

[15] Ulmer, W. and Matsinos, E. (2010) Theoretical Methods for the Calculation of 
Bragg Curves and 3D Distribution of Proton Beams. European Physical Journal Special Topics, 190, 1-81. https://doi.org/10.1140/epjst/e2010-01335-7

[16] Ulmer, W. and Carabe-Fernandez, A. (2017) Aspects of Physical Principles of the Proton Therapy with Inclusion of Nuclear Interactions. Journal of Proton Therapy, 3, Article No. 311.

[17] CERN (2005) GEANT 4 Documents 2005. http://GEANT4.web.cern/GEANT4/G4UsersDocuments/Overview/html/

[18] Ulmer, W. (2012) On the Creation of High Energy Bremsstrahlung and Intensity by a Multitarget and repeated Focusing of the Scattered Electrons by Small-Angle Backscatter at the Wall of a Cone and Magnetic Fields-A Possible Way to Improve Linear Accelerators in Radiotherapy and to Verify Heisenberg-Euler Scatter. Radiation Physics and Chemistry, 81, 387-402.

https://doi.org/10.1016/j.radphyschem.2011.12.033

[19] Ulmer, W. (2013) Creation of High Energy/Intensity Bremsstrahlung by a Multi-Target and Focusing of the Scattered Electrons by Small-Angle Backscatter at a Cone Wall and a Magnetic Field-Enhancement of the Outcome of Linear Accelerators in Radiotherapy. International Journal of Medical Physics, Clinical Engineering and Radiation Oncology, 2, 147-160. https://doi.org/10.4236/ijmpcero.2013.24020

[20] Ulmer, W., Pyyry, J. and Kaissl, W. (2005) A 3D Photon Superposition/Convolution Algorithm and Its Foundation on Results of Monte Carlo Calculations. Physics in Medicine \& Biology, 50, 1767-1790. https://doi.org/10.1088/0031-9155/50/8/010

[21] VARIAN (2008) VARIAN Medical Systems-User Guide: TrueBeam ${ }^{\oplus}$ Radiotherapy System-Medical Linear Accelerator Developed by Varian Medical System, Linear Accelerator Guide, SIEMENS Helthineers-VARIAN. Varian Inc., Palo Alto.

[22] Welsh, J.S., Lock, M., Harari, P.M., Tomé, W.A., Fowler, J., Mackie, T.R., Ritter, M., Kapatoes, J., Forrest, L., Chappell, R., Paliwal, B. and Mehta, M.P. (2006) Clinical Implementation of Adaptive Helical Tomotherapy: A Unique Approach to Image-Guided Intensity-Modulated Radiotherapy. Technology in Cancer Research \& Treatment, 5, 465-479. https://doi.org/10.1177/153303460600500503

[23] Heisenberg, W. and Euler, H. (1936) Folgerungen aus der Diracschen Theorie des Positrons. Zeitschrift für Physik, 98, 714-732. https://doi.org/10.1007/BF01343663

[24] Feynman, R.P. (1972) Photon-Hadron Interaction. CRC Press, Boca Raton.

[25] Molière, G. (1955) Multiple Scatter of Charged Particles Passing through Matter. Zeitschrift für Naturforschung A, 10, 177-211.

https://doi.org/10.1515/zna-1955-0301

[26] Klein, S.R. (2001) Electrodynamics at the Highest Energies. arXiv: hep/0112018v1.

[27] Schälicke, A., Ivanchenko, V., Maire M. and Urban, L. (2008) Improved Description of Bremsstrahlung for High-Energy Electrons in Geant4. 2008 IEEE Nuclear Science Symposium Conference Record, Dresden, 19-25 October 2008, 2788-2791. https://doi.org/10.1109/NSSMIC.2008.4774950

[28] Svensson, R. and Brahme, A. (1996) Effective Source Size, Yield and Beam Profile from Multi-Layered Bremsstrahlung Targets. Physics in Medicine \& Biology, 41, 1353-1379. https://doi.org/10.1088/0031-9155/41/8/008

[29] Friedrich, R., Peinke, R., J. and Renner, C. (2000) How to Quantify Deterministic and Random Influences on the Statistics of the Foreign Exchange Market. Physical Review Letters, 84, 5224-5227. https://doi.org/10.1103/PhysRevLett.84.5224

[30] Vincent, P.H. (1985) An Introduction to Signal Detection and Estimation. Sprin- 
ger-Verlag, New York, 141-204.

[31] Ulmer, W., Cornelissen, G. and Halberg, F. (2014) Interaction among (Quantum Mechanical) Resonance-Coupled Electromagnetic Circuits Relevant to a Natural Week. World Heart Journal, 4, 35-70.

[32] Tsu, R. and Datta, T. (2008) Conductance and Wave Impedance of Electrons. Electromagnetics Research Symposium, Hangzhou, March 2008, 24-28.

[33] Luryi, S. (1988) Quantum Capacitance Device. Applied Physics Letters, 52, Article No. 501. https://doi.org/10.1063/1.99649

[34] Yakymakha, O.L. and Kalnibolotskij, Y.M. (1994) Very-Low-Frequency Resonance of MOSFET Amplifier Parameters. Solid-State Electronics, 37, 1739-1751. https://doi.org/10.1016/0038-1101(94)90221-6 


\section{Appendix: Formula for the Electron Spectrum Leaving the Accelerator Tube}

Formulas (19), (20) have been developed by the present author with regard to some problems of describing the electron beams in Monte-Carlo calculations with GEANT 4 [17], which appeared in connection with ref. [20], but they have never been published before.

Definitions: The energy $E$ referring to the spectral distribution and the nominal energy $E_{0}$ only refer to the kinetic energy of electrons, their total energy results by the addition of the rest energy $m \cdot c^{2}$. With regard to the following formulas, it must be noted that all terms referring to energy are considered as dimensionless numerical values, i.e., they are divided by a unit energy $E_{\text {unit }}=1$ $\mathrm{MeV}$. The following terms are used in order to obtain the energy spectral distribution $E_{s p}$ :

$$
\begin{aligned}
& \left.\begin{array}{l}
\delta=E_{0}-E ; \quad s=\frac{\delta}{2 \cdot \pi} ; \quad \sigma=\frac{\pi}{2} \cdot[1+\operatorname{erf}(s)] \\
\operatorname{erf}(s)=\frac{1}{\sqrt{\pi}} \int_{0}^{s} \mathrm{e}^{-s^{2}} \mathrm{~d} s
\end{array}\right\} \\
& \left.\begin{array}{l}
E_{s p}=\operatorname{erf}\left(E / m c^{2}\right) \cdot \mathrm{e}^{-\frac{\delta^{2}}{\sigma^{2}}} \\
E_{s p}=E_{s p} / \operatorname{Max}\left(E_{s p}\right)
\end{array}\right\}
\end{aligned}
$$

With the help of the operation $E_{s p} / \operatorname{Max}\left(E_{s p}\right)$ the maximum is normalized.

Formulas (19) and (20) and Figure 11 refer to data presented in ref. [20]. However, by checking depth dose curves of different vendors it appears to be justified that the above formulas only need slight modifications to be valid for some different machine types.

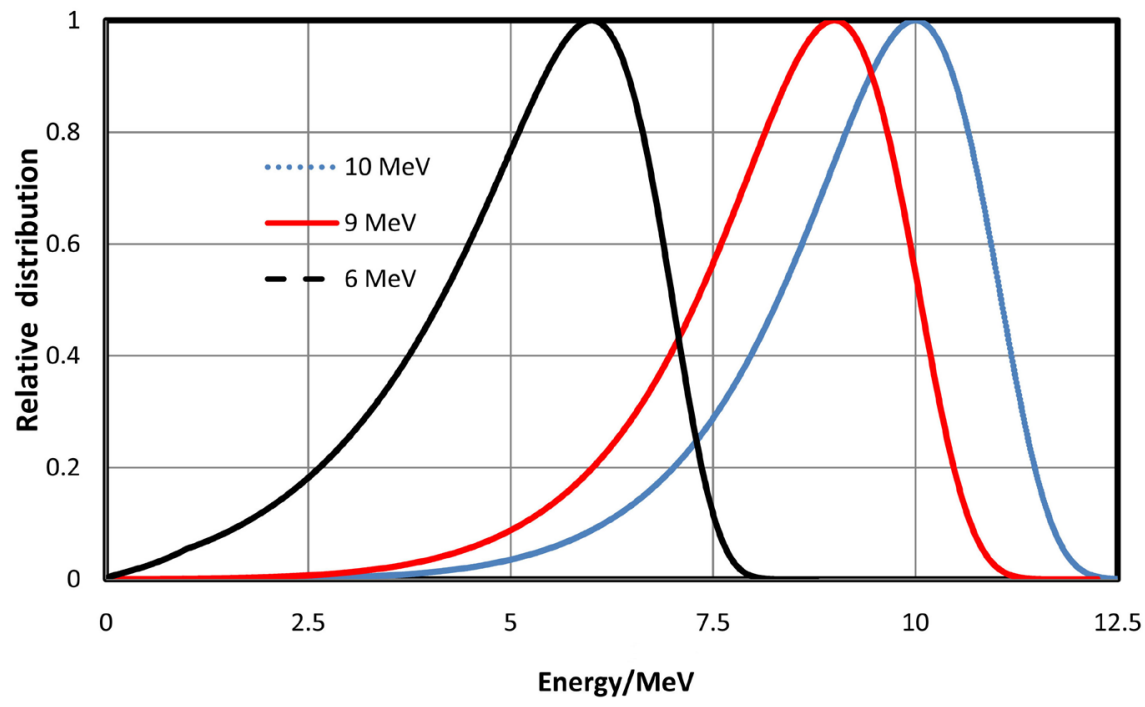

Figure 11. Spectral energy distribution of electron beams and some interesting energies leaving the accelerator tube. 
It should be pointed out that the parameters s, $\sigma$ and $\delta$ in Equation (19) depend on the actual energy E, and, by that, the form of the distribution function is determined. Thereafter, the electron beam is bypassed by a bending magnet, and a slit only permits a narrow Gaussian distribution before it impinges the target. 